\title{
Filter-based stochastic algorithm for global optimization
}

\author{
M. Joseane F. G. Macêdo • Elizabeth W. \\ Karas - M. Fernanda P. Costa • Ana Maria \\ A. C. Rocha
}

Received: date / Accepted: date

\begin{abstract}
We propose the general Filter-based Stochastic Algorithm (FbSA) for the global optimization of nonconvex and nonsmooth constrained problems. Under certain conditions on the probability distributions that generate the sample points, almost sure convergence is proved. In order to optimize problems with computationally expensive black-box objective functions, we develop the FbSA-RBF algorithm based on the general FbSA and assisted by Radial Basis Function (RBF) surrogate models to approximate the objective function. At each iteration, the resulting algorithm constructs/updates a surrogate model of the objective function and generates trial points using a dynamic coordinate search strategy similar to the one used in the Dynamically Dimensioned Search method. To identify a promising best trial point, a non-dominance concept based on the values of the surrogate model and the constraint violation at the trial points is used. Theoretical results concerning the sufficient conditions for the almost surely convergence of the algorithm are presented. Preliminary numerical experiments show that the FbSA-RBF is competitive when compared with other known methods in the literature.
\end{abstract}

Keywords Global optimization · Dynamically dimensioned search · Filter method.

M. Joseane F. G. Macêdo

Dep. of Exact and Natural Sciences, Federal Rural University of Semi-Árido, Mossoró, Brazil E-mail: joseane@ufersa.edu.br

Elizabeth W. Karas

Dep. of Mathematics, Federal University of Paraná, Curitiba, Brazil

E-mail: ewkaras@ufpr.br

M. Fernanda P. Costa

Centre of Mathematics, University of Minho, Campus de Gualtar, Braga, Portugal

E-mail: mfc@math.uminho.pt

Ana Maria A. C. Rocha

ALGORITMI Center, University of Minho, Campus de Gualtar, Braga, Portugal

E-mail: arocha@dps.uminho.pt 


\section{Introduction}

This paper aims to contribute to the research area of global optimization by proposing a general Filter-based Stochastic Algorithm (FbSA) for nonconvex and nonsmooth constrained optimization problems. One of the main goals of this paper is to prove that, under certain hypotheses on the probability distributions that generate the sample points, the algorithm converges to a global optimum of the problem in a probabilistic sense. Since many real-world optimization problems involve black-box objective functions whose objective function values are obtained through computationally expensive simulations, another main goal of this paper is to develop a FbSA assisted by Radial Basis Function (RBF) surrogate models to approximate the objective function to conform to the proposed general FbSA. We denote this algorithm as FbSA-RBF. The mathematical formulation of the problem is

$$
\begin{array}{ll}
\text { minimize } & f(x) \\
\text { subject to } & c_{i}(x) \leq 0, i \in \mathcal{I} \\
& c_{i}(x)=0, i \in \mathcal{E} \\
& x \in \mathcal{D}
\end{array}
$$

where $f: A \subseteq \mathbb{R}^{n} \rightarrow \mathbb{R}$ is a deterministic function defined on a set $A \supseteq \mathcal{D}$ of the $\mathbb{R}^{n}, c_{i}: \mathbb{R}^{n} \rightarrow \mathbb{R}, i \in \mathcal{I} \cup \mathcal{E}$ are the constraint functions, and $\mathcal{D} \subset \mathbb{R}^{n}$ is a finite box of the form $\mathcal{D}=\left\{x \in \mathbb{R}^{n}: l_{i} \leq x_{i} \leq u_{i}, \forall i=1,2, \ldots, n\right\}$, and all lower and upper bounds $l_{i}$ and $u_{i}$ are finite for $i=1, \ldots, n$. The feasible region is denoted by $\mathcal{D}_{V}:=\left\{x \in \mathcal{D}: c_{i}(x) \leq 0, i \in \mathcal{I}, c_{i}(x)=0, i \in \mathcal{E}\right\}$. We assume that $f$ is bounded below, and there exists a global minimizer $x^{*}$ of $f$ over $\mathcal{D}_{V}$ with $f$ continuous at point $x^{*}$. It is also assumed that $f$ and $c_{i}$, with $i \in \mathcal{I} \cup \mathcal{E}$, are Lebesgue measurable.

Many different approaches have been proposed for the global optimization of (1). Among these are the penalty methods, which reformulate (1) to the minimization of an appropriate penalty function over $\mathcal{D}$ [3, 5, 9, 12, 23, 32. Mathematically, the penalty function is defined by the objective function and the constraints violation (penalty terms) multiplied by some positive penalty parameter. However, initializing the penalty parameter and adjusting its value throughout the iterative process are problematic issues since their values affect the performance of the algorithms. In some cases, the optimal solution is obtained when the penalty parameter tends towards infinity.

There are in the literature other penalty functions, namely the exact penalty functions and the augmented Lagrangian functions for which, in general, it is sufficient a finite parameter value to guarantee convergence to the optimal solution of problem (1).

Exact penalty functions and augmented Lagrangian functions have been extended for global optimization (see [6, 12, 19, 47], and references therein). For example, in [12, an Exact Penalty Global Optimization (EPGO) algorithm is proposed, where an improved version of the deterministic DIRECT algorithm [18] is used to globally solve a finite sequence of bound constrained subproblems. The bound constrained subproblems are defined by a non-differentiable exact penalty function for given values of the penalty parameter and an automatic updating rule of the penalty parameter that occurs only a finite number of times. It is proved that EPGO produces a sequence of optimal solutions of the underlying subprob- 
lems such that any limit point of the sequence is a global solution of the related constrained problem.

Other strategies for handling the constraints of the problem, that avoid updating the penalty parameters associated to the penalty functions, have been proposed in the literature. For instance, techniques based on multi-objective concepts in which the constrained problem is reformulated as a bi/multi-objective one, being the objective function and the infeasibility measures minimized simultaneously [1, 2,17. One of the most known is the filter method introduced by Fletcher and Leyffer in [17, which guarantee sufficient progress towards feasibility and optimality of nonlinear constrained problems. The filter method uses the concept of dominance, borrowed from multi-objective optimization, to build a filter that accepts iterates if they improve the objective function or improve a constraint violation function based on the Pareto dominance rule. Ever since, an abundance of filter-based approaches have been proposed [4, 10, 11, 14, 15, 21, 31, 46, 48.

In particular, the filter methods have been also incorporated into stochastic algorithms [24, 28, 34, 42, and the resulting algorithms have been shown that the filter methodology is promising when compared to other strategies. For example, in 42, a filter methodology is incorporated into the population-based artificial fish swarm (P-BF AFS) algorithm for globally solving nonsmooth constrained optimization problems. The constrained problem is replaced by a sequence of bound constrained bi-objective subproblems. The P-BF AFS is used to solve the bound constrained bi-objective subproblems with an increasing accuracy required. The filter methodology is used to accept non-dominated trial points that improve over the current ones.

In 28 is presented a method that combines the filter method and the Dynamically Dimensioned Search (DDS) method [45], for solving nonsmooth constrained global optimization problems. The proposed algorithm reformulates the constrained optimization problem as a bound constrained bi-objective one and uses the filter method to control the progress related to optimality and feasibility by defining a forbidden region of points using the flat or slanting filter rule [28]. At each iteration, the algorithm computes a set of trial points using a similar strategy to DDS to explore the search space for a global optimum. The filter methodology is used to accept the best non-dominated trial point that improves related to the best current one. The resulting algorithm is refereed as A2-FF or A2-SF if it is used the flat or slanting filter rule, respectively. The A2-FF/SF is enriched with a poll-strategy which is invoked whenever a best non-dominated point is not found. This procedure searches in a vicinity of the least infeasible non-dominated point found so far, with the hope to approach a different part of the feasible region of the problem.

It is noteworthy that the DDS is a stochastic global algorithm [45] developed for solving calibration problems that arise in the context of watershed simulation models. These problems are bound constrained ones, with many parameters/variables and computationally expensive objective functions. At each iteration, the DDS generates a randomly trial point by perturbing some coordinates of the current best point, which are dynamically and probabilistically chosen. At the beginning, the DDS searches globally, and becomes a more local search as the number of functions evaluations approaches the maximum allowed. The transition from global to local search is achieved by dynamically and probabilistically reducing the number of dimensions to be perturbed in the neighborhood of the current best solution. 
Since DDS has shown to be a simple and robust tool for solving computationally expensive calibrations problems, a variety of new approaches or extensions that use a dynamic coordinate search strategy similar to the one used in DDS have been proposed in the literature [36, 37, 38, 39, 44, For example, in [44, the DDS algorithm was modified to solve discrete, single-objective, constrained water distribution system design problems. The ConstrLMSRBF algorithm for large-scale optimization involving expensive black-box objective and constraint functions is proposed in 36. This algorithm is an extension of the Local Metric Stochastic RBF (LMSRBF) algorithm 38 designed for bound constrained optimization problems with an expensive black-box objective function. The ConstrLMSRBF uses RBF surrogate models for the objective function and also for all the constraint functions. The ConstrLMSRBF requires a feasible starting point and it always attempts to maintain feasibility along the entire iterative process.

In this paper, we aim to propose a general FbSA for solving nonconvex and nonsmooth constrained global optimization problems. This study comes in sequence of our previous preliminary practical study concerning the filter and DDS methods 28. Under certain conditions on the probability distributions that generate the points, almost sure convergence is proved for a class of algorithms conforming to this general algorithm. In order to solve problems with computationally expensive black-box objective functions, a FbSA assisted by Radial Basis Function surrogate models to approximate the objective function, according to the general FbSA, is presented.

The paper is organized as follows. In Section 2 the general FbSA is presented and its almost sure convergence is established. Section 3 describes the FbSA-RBF assisted by surrogate models to approximate the objective function, and presents its convergence analysis. Section 4 shows the numerical results and the paper is concluded in Section 5 .

\section{General filter-based stochastic algorithm}

In this section, we present the general FbSA for solving nonconvex and nonsmooth constrained global optimization problem (1). The framework of the embedded filter algorithm is similar to [25, Alg. 2.1]. Due to stochastic nature of the algorithm, the iterates are treated as random vectors whose realizations are in $\mathcal{D}$, following the ideas of [35]. The algorithm is general since the iterates are given randomly by any probability distribution. The global convergence of the FbSA in the probabilistic sense is established.

\subsection{The algorithm}

The general FbSA generates two sequences of random vectors whose realizations are in $\mathcal{D} \subset \mathbb{R}^{n}$. Namely, the sequence of iterates $\left(X_{k}\right)$ and the sequence of the best iterates $\left(X_{k}^{*}\right)$ selected from $\left(X_{k}\right)$.

The sequence of iterates $\left(X_{k}\right)$ is defined on a probability space $(\Omega, \mathcal{B}, P)$, where $\Omega$ is the sample space, $\mathcal{B}$ is a $\sigma$-algebra and $P: \mathcal{B} \rightarrow[0,1]$ is a probability function. The random vector $X_{k}:(\Omega, \mathcal{B}) \rightarrow(\mathcal{D}, \mathcal{B}(\mathcal{D}))$ represents the $k$ th function evaluation 
iterate, where $\mathcal{B}(\mathcal{D})$ are the Borel sets in $\mathcal{D}$. At iteration $k$, to define $X_{k}$, a collection of random elements is used, herein denoted by

$$
\left\{\Lambda_{k_{j}}:(\Omega, \mathcal{B}) \rightarrow\left(\Omega_{k_{j}}, \mathcal{B}_{k_{j}}\right), \text { for } j=1, \ldots, \ell_{k}\right\}
$$

where $\ell_{k}>1$ is the number of random elements that are generated. The FbSA is general in the sense that to define the random vectors $X_{k}$ there exists a certain freedom to choose the probability space $(\Omega, \mathcal{B}, P)$ of the collection of random elements.

To simplify the description of general FbSA, the following notation is used. Given an initial random vector $X_{0} \in \mathcal{D}$, the oracle associated to the collection of random elements is defined as follows: $\mathcal{O}_{0}=\left\{X_{0}\right\}$ and for each iteration $k \geq 1$

$$
\mathcal{O}_{k}=\left\{\Lambda_{t_{j}}: t=1, \ldots, k \text { and } j=1, \ldots, \ell_{k}\right\}=\mathcal{O}_{k-1} \cup\left\{\Lambda_{k_{1}}, \cdots, \Lambda_{k_{\ell_{k}}}\right\}
$$

Hence, in the general FbSA, using a deterministic function $\Theta: \mathcal{O}_{k} \rightarrow \mathcal{D}$, the random vector iterate is computed as $X_{k}=\Theta\left(\mathcal{O}_{k}\right)$ from the random elements of $\mathcal{O}_{k}$. We note that, in general, a realization of $X_{k}$ can fall outside of the domain $\mathcal{D}$. To prevent this, the deterministic function has the property to transform any point of $\mathbb{R}^{n}$ into a suitable point of $\mathcal{D}$. Since $\mathcal{D}$ is compact, the function $\Theta\left(\mathcal{O}_{k}\right)$ can involve a projection into $\mathcal{D}$ whenever $X_{k} \notin \mathcal{D}$.

The progress of the random vector iterates $X_{k}, k \geq 1$, is controlled by a filter methodology as suggested in [8,25. The filter methodology treats the optimization problem (1) as bi-objective, and attempts to minimize both the objective function $f$ and a nonnegative aggregate constraint violation function $h$.

Herein, the constraint violation function $h: \mathbb{R}^{n} \rightarrow \mathbb{R}^{+}$is defined by

$$
h(x)=\left\|c^{+}(x)\right\|,
$$

where $\|\cdot\|$ is an arbitrary norm and $c^{+}: \mathbb{R}^{n} \rightarrow \mathbb{R}^{m}$ is given by

$$
c_{i}^{+}(x)= \begin{cases}c_{i}(x), & \text { if } i \in \mathcal{E} \\ \max \left\{0, c_{i}(x)\right\}, & \text { if } i \in \mathcal{I}\end{cases}
$$

The filter method is an efficient technique that builds the forbidden region of the points, known as dominated region. The filter is defined as a set of pairs $\left\{\left(f_{j}, h_{j}\right)\right\}$ from the former iterations.

The general FbSA deals with the filter and with the forbidden region associated with it, as follows. At iteration $k=0$, given an initial random vector $X_{0} \in \mathcal{D}$, we set $X_{0}^{*}=X_{0}$, and we initialize the filter $F_{k}=\emptyset$ and define the forbidden region $\mathcal{F}_{k}=\emptyset$.

At the beginning of each iteration $k \geq 1$, the pair $\left(f\left(X_{k-1}^{*}\right), h\left(X_{k-1}^{*}\right)\right)$ from the previous best iterate is temporarily added to the current filter, $\bar{F}_{k-1} \equiv F_{k-1} \cup$ $\left\{\left(f\left(X_{k-1}^{*}\right), h\left(X_{k-1}^{*}\right)\right)\right\}$. As proposed in [8], to avoid convergence to infeasible accumulation points $X^{*}$ where $h\left(X^{*}\right)>0$, we add an envelope around the temporary current filter $\bar{F}_{k-1}$, and a pair $(f(x), h(x))$ is non-dominated by any pair of the filter, if for all $\left(f_{j}, h_{j}\right) \in \bar{F}_{k-1}$

$$
f(x)+\alpha h(x) \leq f_{j} \quad \text { or } \quad h(x) \leq(1-\alpha) h_{j}
$$


where $\alpha \in(0,1)$ is a given constant. A filter based on this acceptance rule is called by slanting filter. Taking into account the acceptance rule (4), the forbidden region is defined by

$$
\overline{\mathcal{F}}_{k-1}=\mathcal{F}_{k-1} \cup\left\{x \in \mathcal{D}: f(x)+\alpha h(x) \geq f\left(X_{k-1}^{*}\right) \text { and } h(x) \geq(1-\alpha) h\left(X_{k-1}^{*}\right)\right\} .
$$

Thus, a realization of $X_{k}$ is computed such that the pair $\left(f\left(X_{k}\right), h\left(X_{k}\right)\right)$ is acceptable by the filter $\bar{F}_{k-1}$, or equivalently $X_{k} \notin \overline{\mathcal{F}}_{k-1}$. One must keep in mind that the forbidden region is never constructed in the algorithm, but helps the understanding of the process.

When $X_{k}$ produces a reduction on $f$, the iteration is called an $f$-type iteration. Otherwise, it is called an $h$-type iteration [20,31. This classification is used for updating the filter. Herein, the filter is updated only at $h$-type iterations, in such iterations the pair $\left(f\left(X_{k-1}^{*}\right), h\left(X_{k-1}^{*}\right)\right)$ becomes permanent in the filter $F_{k}$, and all pairs that are dominated by $\left(f\left(X_{k-1}^{*}\right), h\left(X_{k-1}^{*}\right)\right)$ can be removed from the filter. If the filter is not updated, it remains unchanged, and the pair $\left(f\left(X_{k-1}^{*}\right), h\left(X_{k-1}^{*}\right)\right)$ is discarded. Thus, the filter is updated as follows:

$$
F_{k}=\left\{\begin{array}{l}
\bar{F}_{k-1}, \text { if } k \text { is an } h \text {-type iteration } \\
F_{k-1}, \text { otherwise. }
\end{array}\right.
$$

This updating rule prevents the addition of feasible points to the filter. Note that, if $X_{k-1}^{*}$ is feasible then $X_{k}$ must decrease $f$ to be accepted by the filter $F_{k-1} \cup\left\{\left(f\left(X_{k-1}^{*}\right), 0\right)\right\}$. Therefore, the iteration must be an $f$-type iteration, and consequently the pair $\left(f\left(X_{k-1}^{*}\right), 0\right)$ is discarded. Furthermore, this updated rule assures movements towards some infeasible regions to return back later to a different part of the feasible one.

The new iterate $X_{k} \in \mathcal{D}$ accepted by the filter will be selected as a new best point if it does not increase the infeasibility measure more than a small positive quantity $\varepsilon_{h}>0$, from the current best point. Figure 1 illustrates the region of pairs $(f, h)$ that are forbidden or dominated by $\left(f\left(X_{k-1}^{*}\right), h\left(X_{k-1}^{*}\right)\right)$ and the region of pairs that are considered best than it. Allowing a small controlled increase in the infeasibility measure $h$ with a reduction in the optimality measure $f$, this strategy aims to promote the exploration of other potentially promising neighborhood areas of the feasible region and to prevent convergence to optimal local solutions. Note that once a best feasible point is obtained, the best point will be updated only if the new one reduces the objective function value.

A formal description of the general FbSA for solving problem (1) is presented in Algorithm 1

\subsection{Global convergence}

In this section we present the convergence analysis of Algorithm 1 in the probabilistic sense.

The first lemma ensures that all elements of the filter correspond to infeasible points and that the algorithm is well-defined in the sense that whenever the current point does not satisfy the stop criterion, a new not forbidden point can be computed. 
Fig. 1 Filter criterion in $f \times h$ plane
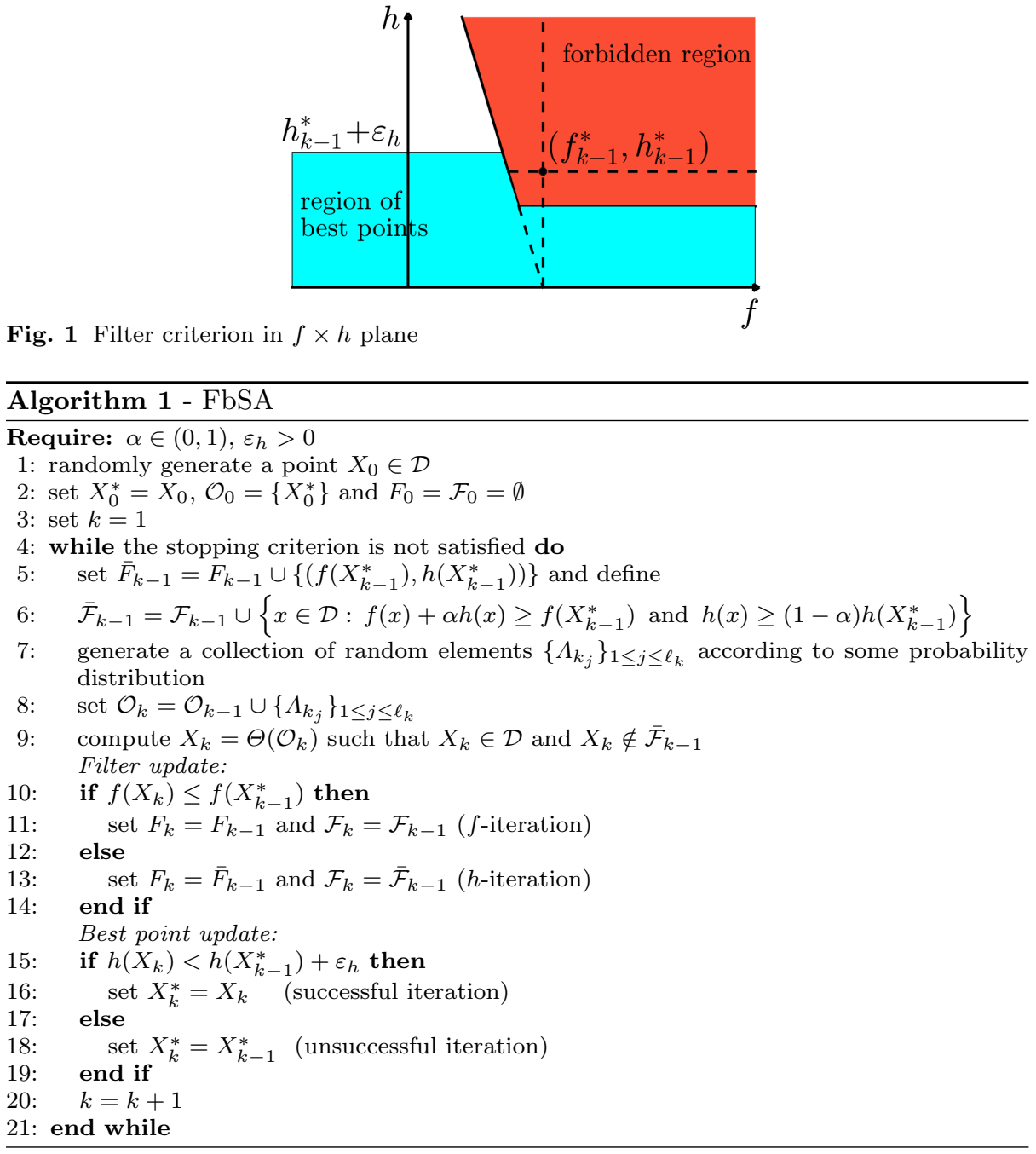

Lemma 1 For all $k \geq 1$ such that $X_{k-1}$ does not satisfy the stopping criterion of Algorithm 1, the following two statements hold:

(i) $h\left(X_{j}\right)>0$, for all $\left(f\left(X_{j}\right), h\left(X_{j}\right)\right) \in F_{k-1}$;

(ii) There exists $X_{k} \notin \overline{\mathcal{F}}_{k-1}$.

Proof Due to the general FbSA framework and the embedded filter methodology that is similar to the one presented in 225, the proof follows analogous to [25, Alg. $2.1]$.

The next theorem states that any accumulation point of the sequences generated by the algorithm is feasible. 
Theorem 1 Consider the sequences $\left(X_{k}\right)$ and $\left(X_{k}^{*}\right)$ generated by Algorithm 1 . Then $h\left(X_{k}\right) \rightarrow 0$ and $h\left(X_{k}^{*}\right) \rightarrow 0$. In addition, the number of unsuccessful iterations is finite.

Proof The result for the sequence $h\left(X_{k}\right)$ follows from [25, Thm. 2.3]. By the selection of the best point in the algorithm,

$$
h\left(X_{k}^{*}\right)=h\left(X_{k}\right) \quad \text { or } \quad h\left(X_{k}^{*}\right)=h\left(X_{k-1}^{*}\right) \leq h\left(X_{k}\right)-\varepsilon_{h}<h\left(X_{k}\right),
$$

where $\varepsilon_{h}>0$. So, $0 \leq h\left(X_{k}^{*}\right) \leq h\left(X_{k}\right)$, for all $k \in \mathbb{N}$, and consequently $h\left(X_{k}^{*}\right) \rightarrow 0$.

Now suppose by contradiction that the number of unsuccessful iterations is infinite. In this case, there exists a subsequence of indices $\left(k_{j}\right)_{j \geq 1}$ satisfying,

$$
h\left(X_{k_{j}}\right) \geq h\left(X_{k_{j}-1}^{*}\right)+\varepsilon_{h} \quad \text { and } \quad h\left(X_{k_{j}}^{*}\right)=h\left(X_{k_{j}-1}^{*}\right) .
$$

Thus, $h\left(X_{k_{j}}\right) \geq h\left(X_{k_{j}}^{*}\right)+\varepsilon_{h}$. As $h\left(X_{k}\right)$ and $h\left(X_{k}^{*}\right)$ converge to zero, setting $j \rightarrow \infty$, it follows that $\varepsilon_{h} \leq 0$, which contradicts the fact that $\varepsilon_{h}$ is a strictly positive constant.

Now we analyse the convergence of Algorithm 1 in the probabilistic sense following the ideas of 35 . We say that an algorithm converges to the global minimum of $f$ on $\mathcal{D}$ in probability, or almost surely, if the sequence $\left(f\left(X_{k}^{*}\right)\right)$ converges to $f^{*}$ in probability, or almost surely.

Let $\sigma\left(\mathcal{O}_{k}\right)$ be the $\sigma$-algebra generated by the oracle which represents all the information that can be derived from the random elements in $\mathcal{O}_{k}$.

In the light of Lemma 1, we shall suppose that Algorithm 1 generates infinite sequences $\left(X_{k}\right)$ and $\left(X_{k}^{*}\right)$. Furthermore, let $\mu$ be the Lebesgue measure in $\mathbb{R}^{n}$ and assume the following hypotheses.

H1 The function $f$ has a global minimizer $x^{*}$ over $\mathcal{D}_{V}$, with $f$ continuous at point $x^{*}$. The functions $f$ and $c_{i}, i \in \mathcal{I} \cup \mathcal{E}$, are Lebesgue measurable.

H2 For all $\rho, \varepsilon>0, \mu\left(T_{\rho, \varepsilon}\right)>0$, where

$$
T_{\rho, \varepsilon}:=\left\{x \in \mathcal{D}: h(x) \leq \rho \text { and } f(x)<f^{*}+\varepsilon\right\} .
$$

H3 For all $\delta>0$,

$$
\psi_{\mathcal{D}}(\delta):=\inf _{z \in \mathcal{D}} \mu(B(z, \delta) \cap \mathcal{D})>0 .
$$

The Hypotheses $\mathrm{H} 1$ and $\mathrm{H} 2$ are satisfied as long as $\mathcal{D}_{V}$ is a bounded region and the functions $f$ and $c_{i}, i \in \mathcal{I} \cup \mathcal{E}$, are continuous. Hypothesis $H 2$ is a key assumption and quite usual in this context [34,42]. It precludes $f$ from having an isolated discontinuous optimum point (for more details, see 22]). In 34, the convergence is guaranteed almost surely for a point in the set $S^{*}=\left\{x \in \mathcal{D}_{V}: f(x) \leq f_{\text {egs }}^{*}\right\}$, where $f_{\text {egs }}^{*}$ is the infimum on all values $f_{t}$ for which

$$
\mu\left(\left\{z \in \mathcal{D}: h(z) \leq \rho, f(z)<f_{t}\right\}\right)>0,
$$

for all $\rho>0$. Hypothesis $H 3$ is also considered in [35] and guarantees that the intersection of $\mathcal{D}$ and any ball centered in a point of $\mathcal{D}$ has positive measure. This condition is important to prove the next lemma. 
Lemma 2 Consider the sequence $\left(X_{k}\right)$ generated by Algorithm 1 , Let $\left(X_{k_{j}}\right)_{j \geq 1}$ be a subsequence of $\left(X_{k}\right)$ such that for each $j \geq 1, X_{k_{j}}$ has a conditional density

$$
g_{k_{j}}\left(x \mid \sigma\left(\mathcal{O}_{\left(k_{j}\right)-1}\right)\right) .
$$

Suppose that

$$
\mu(\{x \in \mathcal{D}: G(x)=0\})=0, \quad \text { where } \quad G(x):=\inf _{j \geq 1} g_{k_{j}}\left(x: \sigma\left(\mathcal{O}_{\left(k_{j}\right)-1}\right)\right) .
$$

Then, the following statements hold:

(i) For all $z \in \mathcal{D}$ and $\delta>0$, there exists $v(z, \delta) \in(0,1)$ such that

$$
P\left(X_{k_{j}} \in B(z, \delta) \cap \mathcal{D} \mid \sigma\left(\mathcal{O}_{\left(k_{j}\right)-1}\right)\right) \geq v(z, \delta) .
$$

(ii) For all $\varepsilon>0$, there exists $L(\varepsilon) \in(0,1)$ such that

$$
P\left(X_{k_{j}} \in \mathcal{D}: f\left(X_{k_{j}}\right)<f\left(x^{*}\right)+\varepsilon \mid \sigma\left(\mathcal{O}_{\left(k_{j}\right)-1}\right)\right) \geq L(\varepsilon) .
$$

Proof (i) Fixed $\delta>0$ and $z \in \mathcal{D}$, by the definition of probability and integration properties we have, for all $j \geq 1$, that

$$
\begin{aligned}
P\left(X_{k_{j}} \in(B(z, \delta) \cap \mathcal{D}) \mid \sigma\left(\mathcal{O}_{\left(k_{j}\right)-1}\right)\right) & =\int_{B(z, \delta) \cap \mathcal{D}} g_{k_{j}}\left(x \mid \sigma\left(\mathcal{O}_{\left(k_{j}\right)-1}\right)\right) d x \\
& \geq \int_{B(z, \delta) \cap \mathcal{D}} G(x) d x .
\end{aligned}
$$

Using the fact that $G$ is non-negative in $\mathcal{D},(6)$ and Hypothesis $H 3$, we have

$$
v(z, \delta):=\int_{B(z, \delta) \cap \mathcal{D}} G(x) d x>0 .
$$

Thus, for all $j \geq 1$, there exists $v(z, \delta) \in(0,1)$ satisfying (7).

(ii) Given $\varepsilon>0$, by the continuity of $f$ in $x^{*}$, there exists $\delta(\varepsilon)>0$ such that if $\left\|x-x^{*}\right\|<\delta(\varepsilon)$, then $\left|f(x)-f\left(x^{*}\right)\right|<\varepsilon$. Consequently, for each $j \geq 1$ we have the following relation between the events,

$$
\begin{aligned}
{\left[X_{k_{j}} \in \mathcal{D}: f\left(X_{k_{j}}\right)<f\left(x^{*}\right)+\varepsilon\right] } & =\left[X_{k_{j}} \in \mathcal{D}:\left|f\left(X_{k_{j}}\right)-f\left(x^{*}\right)\right|<\varepsilon\right] \\
& \supseteq\left[X_{k_{j}} \in \mathcal{D}:\left\|X_{k_{j}}-x^{*}\right\|<\delta(\varepsilon)\right] .
\end{aligned}
$$

Thus,

$$
\begin{aligned}
P\left(X_{k_{j}} \in \mathcal{D}: f\left(X_{k_{j}}\right)<f\left(x^{*}\right)+\varepsilon \mid \sigma\left(\mathcal{O}_{\left(k_{j}\right)-1}\right)\right) & \geq \\
\geq P\left(X_{k_{j}} \in \mathcal{D}:\left\|X_{k_{j}}-x^{*}\right\|<\delta(\varepsilon) \mid \sigma\left(\mathcal{O}_{\left(k_{j}\right)-1}\right)\right) & \geq v\left(x^{*}, \delta(\varepsilon)\right) .
\end{aligned}
$$

Defining $L(\varepsilon)=v\left(x^{*}, \delta(\varepsilon)\right)>0$, we conclude the proof.

Consider the set of $h$-iterations defined by

$$
\mathcal{K}_{a}=\left\{k \in \mathbb{N}:\left(f\left(X_{k-1}^{*}\right), h\left(X_{k-1}^{*}\right)\right) \text { is added to the filter }\right\} .
$$

Now we present a sufficient condition that guarantees the convergence of Algorithm 1 in terms of the infimum of the conditional densities of the random vector iterates. 
Theorem 2 Consider the sequence $\left(X_{k}^{*}\right)$ generated by Algorithm 1 . Suppose that there exists a subsequence $\left(X_{k_{j}}^{*}\right)$ of $\left(X_{k}^{*}\right)$ such that for each $j \geq 1, X_{k_{j}}^{*}$ has a conditional density $g_{k_{j}}\left(x \mid \sigma\left(\mathcal{O}_{\left(k_{j}\right)-1}\right)\right)$ satisfying (6). Then, the following statements hold:

(i) The sequence $\left(f\left(X_{k}^{*}\right)\right)$ has an accumulation point that is the global minimum of $f$ over $\mathcal{D}_{V}$ almost surely.

(ii) If $\mathcal{K}_{a}$ is finite, then any accumulation point of the sequence $\left(f\left(X_{k}^{*}\right)\right)$ is the global minimum of $f$ over $\mathcal{D}_{V}$ almost surely, this is, $f\left(X_{k}^{*}\right) \rightarrow f^{*}$ almost surely.

(iii) If $x^{*}$ is unique, then $X_{k}^{*} \rightarrow x^{*}$ almost surely.

Proof (i) Consider the decreasing sequences $\left(\rho_{\ell}\right),\left(\varepsilon_{\ell}\right)$ of positive terms, converging to zero. By Hypothesis $\mathrm{H} 2$ we have that $\mu\left(T_{\rho_{\ell}, \varepsilon_{\ell}}\right)>0$, for all $\ell \geq 1$. Thus, the probability that a point randomly generated in $\mathcal{D}$ belongs to $T_{\rho_{\ell}, \varepsilon_{\ell}}$ is strictly positive. Using this and the hypothesis about the subsequence $\left(X_{k_{j}}^{*}\right)$, by Lemma 2 , item $(i)$, we have for all $\delta>0$ and $z \in \mathcal{D}$, there exists $v(z, \delta) \in(0,1)$ satisfying,

$$
P\left(X_{k_{j}}^{*} \in\left(B(z, \delta) \cap T_{\rho_{\ell}, \varepsilon_{\ell}}\right) \mid \sigma\left(\mathcal{O}_{\left(k_{j}\right)-1}\right)\right) \geq v(z, \delta) .
$$

Thus, for all $\ell \geq 1$, there exists $k_{j_{\ell}}$ such that $X_{k_{j_{\ell}}}^{*} \in T_{\rho_{\ell}, \varepsilon_{\ell}}$ almost surely. Defining $S_{\varepsilon}:=\left\{x \in \mathcal{D}: f(x)<f^{*}+\varepsilon\right\}$, we have $T_{\rho_{\ell}, \varepsilon_{\ell}} \subset S_{\varepsilon_{\ell}}$ and, consequently, $X_{k_{j_{\ell}}}^{*} \in S_{\varepsilon_{\ell}}$ almost surely.

As the sequence $\left(\varepsilon_{\ell}\right)$ is decreasing and converges to zero, given $\varepsilon>0$, there exists $\bar{\ell} \in N$, such that $\varepsilon>\varepsilon_{\bar{\ell}}>\ldots>\varepsilon_{\ell}>\ldots$, for all $\ell \geq \bar{\ell}$, which implies $S_{\varepsilon} \supseteq S_{\varepsilon_{\bar{\ell}}} \supseteq \ldots \supseteq S_{\varepsilon_{\ell}} \supseteq \ldots$ Therefore, $X_{k_{j_{\ell}}}^{*} \in S_{\varepsilon}$ for all $\ell \geq \bar{\ell}$. Consequently,

$$
X_{k_{j_{\ell}}}^{*} \notin S_{\varepsilon} \Rightarrow X_{k_{j_{1}}}^{*} \notin S_{\varepsilon}, X_{k_{j_{2}}}^{*} \notin S_{\varepsilon}, \cdots X_{k_{j_{\ell}}}^{*} \notin S_{\varepsilon} .
$$

Applying Lemma 2, item $(i i)$, there exists $L(\varepsilon) \in(0,1)$ such that for all $j \geq 1$,

$$
P\left(X_{k_{j}}^{*} \in \mathcal{D}: f\left(X_{k_{j}}^{*}\right)<f\left(x^{*}\right)+\varepsilon \mid \sigma\left(\mathcal{O}_{\left(k_{j}\right)-1}\right)\right) \geq L(\varepsilon) .
$$

Rewriting 111 in terms of the index $k_{j_{\ell}}$ and the set $S_{\varepsilon}$, we have

$$
P\left(X_{k_{j_{\ell}}}^{*} \in S_{\varepsilon} \mid \sigma\left(\mathcal{O}_{\left(k_{j_{\ell}}\right)-1}\right)\right) \geq L(\varepsilon)
$$

On the other hand,

$$
\begin{aligned}
P\left(X_{k_{j_{1}}}^{*} \notin S_{\varepsilon}, \ldots, X_{k_{j_{\ell}}}^{*} \notin S_{\varepsilon}\right) & =\prod_{i=1}^{\ell} P\left(X_{k_{j_{i}}}^{*} \notin S_{\varepsilon} \mid X_{k_{j_{1}}}^{*} \notin S_{\varepsilon}, \ldots, X_{k_{j_{(i-1)}}}^{*} \notin S_{\varepsilon}\right) \\
& =\prod_{i=1}^{\ell}\left(1-P\left(X_{k_{j_{i}}}^{*} \in S_{\varepsilon} \mid X_{k_{j_{1}}}^{*} \notin S_{\varepsilon}, \ldots, X_{k_{j_{(i-1)}}}^{*} \notin S_{\varepsilon}\right)\right) .
\end{aligned}
$$

From the conditioning of the random elements in $\mathcal{O}_{k_{j_{i}}-1}$ and 12 ,

$$
P\left(X_{k_{j_{i}}}^{*} \in S_{\varepsilon} \mid X_{k_{j_{1}}}^{*} \notin S_{\varepsilon}, \ldots, X_{k_{j_{(i-1)}}^{*}} \notin S_{\varepsilon}\right) \geq L(\varepsilon) .
$$

Therefore, by (13) and (14), it follows that

$$
P\left(X_{k_{j_{1}}}^{*} \notin S_{\varepsilon}, \ldots, X_{k_{j_{\ell}}}^{*} \notin S_{\varepsilon}\right) \leq(1-L(\varepsilon))^{\ell} .
$$


Thus, for each $\ell \geq 1$, we have

$$
\begin{aligned}
0 & \leq P\left(f\left(X_{k_{j_{\ell}}}^{*}\right)-f^{*} \geq \varepsilon\right)=P\left(f\left(X_{k_{j_{\ell}}}^{*}\right) \geq f^{*}+\varepsilon\right)=P\left(X_{k_{j_{\ell}}}^{*} \notin S_{\varepsilon}\right) \leq \\
& \leq P\left(X_{k_{j_{1}}}^{*} \notin S_{\varepsilon}, \ldots, X_{k_{j_{\ell}}}^{*} \notin S_{\varepsilon}\right) \leq(1-L(\varepsilon))^{\ell} .
\end{aligned}
$$

Consequently,

$$
\lim _{\ell \rightarrow \infty} P\left(f\left(X_{k_{j_{\ell}}}^{*}\right)-f^{*} \geq \varepsilon\right)=0
$$

which means that $f\left(X_{k_{j_{\ell}}}^{*}\right) \rightarrow f^{*}$ in probability. Hence, by a standard result in probability theory [[40], Thm 6.3.1(b)] we have that $f\left(X_{k_{j_{\ell(i)}}^{*}}^{*}\right) \rightarrow f^{*}$ almost surely, with $i \rightarrow \infty$ for some subsequence of index $\left(k_{j_{\ell(i)}}\right)_{i \geq 1}$.

(ii) Now, suppose that $\mathcal{K}_{a}$ is finite. By the filter update rule, the number of iterations in which the objective function increases is finite. Consequently, there exists $\bar{k} \in \mathbb{N}$ such that the sequence $\left(f\left(X_{k}^{*}\right)\right)_{k \geq \bar{k}}$ is non-increasing. On the other hand, given that $f^{*}>-\infty$, we have $\lim _{k \rightarrow \infty} f\left(X_{k}^{*}(\omega)\right)$ exists for each underlying sample point. Thus, by the uniqueness of the limit, we can conclude that

$$
f\left(X_{k}^{*}\right) \rightarrow f^{*} \text { a.s. (almost surely) }
$$

(iii) Given $\varepsilon>0$, define $\tilde{f}:=\inf _{x \in \mathcal{D},\left\|x-x^{*}\right\| \geq \varepsilon} f(x)$. By the uniqueness of $x^{*}$, it follows $\tilde{f}>f^{*}$. From $(16)$, we have that such convergence occurs less than in a set of Lebesgue measure zero; i.e, there exists $\mathcal{N} \subset \Omega$ with $P(\mathcal{N})=0$ and such that $f\left(X_{k}^{*}(\omega)\right) \rightarrow f\left(x^{*}\right)$ for all $\omega \in \mathcal{N}^{c}$. Thus, for all $\omega \in \mathcal{N}^{c}$ there exists $N \in \mathbb{N}$ such that for all $k \geq N$,

$$
f\left(X_{k}^{*}(\omega)\right)-f\left(x^{*}\right)=\left|f\left(X_{k}^{*}(\omega)\right)-f\left(x^{*}\right)\right|<\tilde{f}-f\left(x^{*}\right),
$$

which implies,

$$
f\left(X_{k}^{*}(\omega)\right)<\tilde{f}
$$

for all $k \geq N$ and $\omega \in \mathcal{N}^{c}$.

Now, let us show that $\left\|X_{k}^{*}(\omega)-x^{*}\right\|<\varepsilon$, for all $\omega \in \mathcal{N}^{c}$. In fact, suppose by contradiction that there exists $\omega \in \mathcal{N}^{c}$ such that $\left\|X_{k}^{*}(\omega)-x^{*}\right\| \geq \varepsilon$. By the definition of $\tilde{f}$, it follows that $f\left(X_{k}^{*}(\omega)\right) \geq \tilde{f}$, which contradicts (17). Hence, we have that for all $\omega \in \mathcal{N}^{c}$, there exists $N \in \mathbb{N}$ such that, for all $k \geq N,\left\|X_{k}^{*}(\omega)-x^{*}\right\|<\varepsilon$. Therefore, $X_{k}^{*}(\omega) \rightarrow x^{*}$ a.s.

Next theorem ensures the convergence in probability of Algorithm 1 when a subsequence of the iterates is given as the sum of the best point obtained so far with random vectors with multivariate normal distribution.

Theorem 3 Consider the sequence $\left(X_{k}^{*}\right)$ generated by Algorithm 1 and suppose that there exists a subsequence $\left(X_{k_{j}}^{*}\right)_{j \geq 1}$ such that, for each $j \geq 1$,

$$
X_{k_{j}}^{*}=X_{\left(k_{j}\right)-1}^{*}+W_{j},
$$

where $W_{j}$ is a random vector which conditional distribution $\sigma\left(\mathcal{O}_{\left(k_{j}\right)-1}\right)$ is a multivariate normal distribution with mean vector 0 and covariance matrix $V_{j}$. For each $j \geq 1$, let $\bar{\lambda}_{j}$ be the smallest eigenvalue of $V_{j}$. If $\inf _{j \geq 1} \bar{\lambda}_{j}>0$, then the sequence $f\left(X_{k}^{*}\right)$ has an 
accumulation point that is the global minimum of $f$ over $\mathcal{D}_{V}$ almost surely. In addition, if $\mathcal{K}_{a}$ is finite, then $f\left(X_{k}^{*}\right) \rightarrow f^{*}$ almost surely. Finally, if $x^{*}$ is the unique global minimizer, then $X_{k}^{*} \rightarrow x^{*}$ almost surely.

Proof By the mechanism of the algorithm,

$$
X_{\left(k_{j}\right)-1}^{*}=\sum_{i=1}^{\left(k_{j}\right)-1} X_{i} 1_{E}\left(X_{i}\right),
$$

where $1_{E}$ is the indicator function and $E$ is the event defined by

$$
\begin{aligned}
E= & {\left[h\left(X_{\ell}\right)<h\left(X_{t}\right)+\varepsilon_{h}, \text { for all } t=1, \ldots,\left(k_{j}\right)-1 \text { and } \ell\right. \text { is the smallest index }} \\
& \text { of the points non-dominated by the filter } \left.\overline{\mathcal{F}}_{t} \text { with this property }\right] .
\end{aligned}
$$

For each $i=1, \ldots,\left(k_{j}\right)-1, X_{i}$ is obtained by a deterministic function of the random elements in $\mathcal{O}_{i}$. Consequently, $X_{\left(k_{j}\right)-1}^{*}$ is obtained by a deterministic function of the random elements in $\mathcal{O}_{\left(k_{j}\right)-1}$. By (18), the conditional distribution of $X_{k_{j}}$, given $\sigma\left(\mathcal{O}_{\left(k_{j}\right)-1}\right)$, is multivariate normal distribution, with mean vector $X_{\left(k_{j}\right)-1}^{*}$ and covariance matrix $V_{j}$. This matrix is invertible, for all $j \geq 1$, since by hypothesis $\inf _{j \geq 1} \bar{\lambda}_{j}>0$. Thus, $X_{k_{j}}$ has conditional density given by

$$
g_{k_{j}}\left(x \mid \sigma\left(\mathcal{O}_{\left(k_{j}\right)-1}\right)\right)=\gamma\left[\operatorname{det}\left(V_{j}\right)\right]^{-1 / 2} \Psi\left(\left(x-X_{\left(k_{j}\right)-1}^{*}\right)^{T} V_{j}^{-1}\left(x-X_{\left(k_{j}\right)-1}^{*}\right)\right),
$$

where $\Psi(x)=e^{-x / 2}$ and $\gamma=(2 \pi)^{-n / 2}$

From the definition of the Euclidean norm and of $\bar{\lambda}_{j}$, it follows that

$$
\left\|V_{j}^{-1}\right\|=\sqrt{\text { largest eigenvalue of }\left(V_{j}^{-1}\right)^{T} V_{j}^{-1}}=\sqrt{\frac{1}{\bar{\lambda}_{j}^{2}}}=\frac{1}{\left|\bar{\lambda}_{j}\right|}=\frac{1}{\bar{\lambda}_{j}} .
$$

Using this, the fact that $V_{j}$ is definite positive and the Cauchy-Schwarz inequality, we have, for each $j \geq 1$ and $x \in \mathcal{D}$,

$$
\left(x-X_{\left(k_{j}\right)-1}^{*}\right)^{T} V_{j}^{-1}\left(x-X_{\left(k_{j}\right)-1}^{*}\right) \leq\left\|x-X_{\left(k_{j}\right)-1}^{*}\right\|^{2}\left\|V_{j}^{-1}\right\| \leq \frac{D^{2}}{\bar{\lambda}_{j}},
$$

where $D=\operatorname{diam}(\mathcal{D})=\sup _{x, y \in \mathcal{D}}\|x-y\|<\infty$, once that $\mathcal{D}$ is bounded. As $\Psi$ is monotonically non-increasing,

$$
\Psi\left(\left(x-X_{\left(k_{j}\right)-1}^{*}\right)^{T} V_{j}^{-1}\left(x-X_{\left(k_{j}\right)-1}^{*}\right)\right) \geq \Psi\left(\frac{D^{2}}{\bar{\lambda}_{j}}\right) .
$$

Thus, $\operatorname{det}\left(V_{j}\right) \leq\left(\lambda_{j}^{*}\right)^{n}$, where $\lambda_{j}^{*}$ is the largest eigenvalue of $V_{j}$. Using this and (21) in 20) and the fact that $\Psi$ is monotonically non-increasing we have, for each $x \in \mathcal{D}$,

$$
g_{k_{j}}\left(x \mid \sigma\left(\mathcal{O}_{\left(k_{j}\right)-1}\right)\right) \geq \gamma\left(\lambda_{j}^{*}\right)^{-n / 2} \Psi\left(\frac{D^{2}}{\bar{\lambda}_{j}}\right) \geq \gamma\left(\sup _{\ell \geq 1} \lambda_{\ell}^{*}\right)^{-n / 2} \Psi\left(\frac{D^{2}}{\inf _{\ell \geq 1} \bar{\lambda}_{\ell}}\right)
$$


Thus, for all $x \in \mathcal{D}$,

$$
G(x)=\inf _{j \geq 1} g_{k_{j}}\left(x \mid \sigma\left(\mathcal{O}_{\left(k_{j}\right)-1}\right)\right) \geq \gamma\left(\sup _{\ell \geq 1} \lambda_{\ell}^{*}\right)^{-n / 2} \Psi\left(\frac{D^{2}}{\inf _{\ell \geq 1} \bar{\lambda}_{\ell}}\right)>0,
$$

which implies

$$
\mu(\{x \in \mathcal{D}: G(x)=0\})=0 .
$$

Consequently, the hypotheses of Theorem 2 hold and we get the results.

Therefore, any filter-based stochastic algorithm with the same general structure as Algorithm 1, satisfying the sufficient conditions studied in this section almost surely converges to a solution of the problem.

\section{The algorithm with surrogate models}

In this section, we specify the steps of Algorithm 1 considering Radial Basis Function surrogate models to approximate the expensive objective function of problem (1). We refer the resulting algorithm as FbSA-RBF.

The iterates are computed by a dynamic coordinate search strategy similar to the one used in DDS [45]. For an efficient exploration of the search space, at each iteration $k, n_{t}$ random trial points are generated by adding random perturbations with normal distribution in the dynamically chosen coordinates of the best current point. Then, the best trial point among the $n_{t}$ trial points is selected, that can replace or not, the current best point. In order to reduce the number of function evaluations, a radial basis function interpolation model is used to predict the objective function evaluation in the trial points.

\subsection{Description of the algorithm}

This section describes the main choices and steps of FbSA-RBF algorithm.

\subsubsection{Initial points}

The algorithm computes a set of $n_{0} \geq n+1$ initial points:

$$
I=\left\{\left(X_{j}\right) \in \mathcal{D}: X_{j}=\ell+\beta_{j}(u-\ell), \quad \text { with } \quad 1 \leq j \leq n_{0}\right\}
$$

where $\beta_{j} \in[0,1]^{n}$ is generated by using a symmetric Latin hypercube design, as proposed in [49]. Then, the best initial point $X_{0}^{*} \in I$ is selected as follows.

Definition 1 The initial point $X_{0}^{*}$ is the point of the set $I$ which satisfies one of the following two conditions:

(i) if all points in $I$ are infeasible, then the best initial point will be the one with the smallest infeasibility measure. In case of a tie, select the point with the smallest objective function value;

(ii) if the set $I$ contains feasible points, then the best initial point will be the feasible trial point with the smallest objective function value. 


\subsubsection{Trial points}

At each iteration $k$, the algorithm generates $n_{t}$ trial points by perturbing some or all components of the current best point. The collection of random elements used for computing the trial points is given by

$$
\left\{\Lambda_{k_{j}}\right\}_{1 \leq j \leq 3 n_{t}+1}=\left\{P_{k}, Z_{k}^{t}, \xi_{k}^{t}, i_{k}^{t}\right\},
$$

where $P_{k}$ is the probability of perturbing the coordinates; $Z_{k}^{t}$ is the vector associated to $t$-th trial point, whose components are random numbers uniformly distributed in the interval $[0,1]$, and that with the probability $P_{k}$ will decide to perturb or not certain component of the best current point; $\left(\xi_{k}^{t}\right)_{i} \sim N\left(0,\left(\lambda_{k-1}\right)_{i}^{2}\right)$ is related to the step size as a perturbation to the component $i$ of the current best point, chosen to be perturbed, with a normal distribution with mean 0 and standard deviation $\left(\lambda_{k-1}\right)_{i}$ and $i_{k}^{t}$ is the index associated to the component that can be perturbed.

For each $t=1, \ldots, n_{t}$, we define the set of indices of the coordinates that will be perturbed at the iteration $k$, by

$$
J_{k}^{t}= \begin{cases}\left\{i_{k}^{t}\right\}, & \text { if }\left(Z_{k}^{t}\right)_{i}>P_{k}, \text { for all } i=1, \ldots, n \\ \left\{i:\left(Z_{k}^{t}\right)_{i} \leq P_{k}\right\}, & \text { otherwise. }\end{cases}
$$

The step size $\left(\xi_{k}^{t}\right)_{i}$ is adjusted by controlling the standard deviation $\lambda_{k}$, associated with the magnitude of the step perturbation. As this parameter is related to the control of the randomness or, equivalently, of the diversity of the points generated, it can be increased or decreased depending of the number of success or unsuccessful consecutive iterations.

The deterministic function $\Theta^{t}: \mathcal{O}_{k} \rightarrow \mathcal{D}$ computes the trial points as a perturbation of the current best point as

$$
\left(X_{k}^{t}\right)_{i}=\left\{\begin{array}{cc}
\min \left\{u_{i}, \max \left\{\left(X_{k-1}^{*}\right)_{i}+\left(\xi_{k}^{t}\right)_{i}, \ell_{i}\right\}\right\}, & \text { if } i \in J_{k}^{t} \\
\left(X_{k-1}^{*}\right)_{i}, & \text { if } i \notin J_{k}^{t}
\end{array}\right.
$$

for all $t=1, \ldots, n_{t}$ and $i=1, \ldots, n$.

\subsubsection{Radial basis functions}

Surrogate models are computationally inexpensive approximations that are used to reduce the high cost of the objective function evaluations involved in the optimization process [7,39]. Since $f$ is expensive, we use a surrogate model to approximate $f$ across a RBF model 33 39, briefly described below.

Consider the set $\mathcal{A}_{k-1}=\left\{X_{1}, \ldots, X_{q}\right\} \subset \mathbb{R}^{n}$ of sample points where the values of the objective function $f\left(X_{1}\right), \ldots, f\left(X_{q}\right)$ are known. The RBF model approximates the objective function through the following interpolation function,

$$
S_{k}(X)=\sum_{X_{i} \in \mathcal{A}_{k-1}} \eta_{i} \phi\left(\left\|X-X_{i}\right\|\right)+p(X),
$$

where $\eta_{i} \in \mathbb{R}$, for $i=1, \ldots, q,\|\cdot\|$ is the Euclidean norm, $p$ is a linear function in $\mathbb{R}^{n}$ and the function $\phi$ has the cubic form, $\phi(y)=y^{3}$, as suggested in 39. Note 
that other functions can be used, for example $\phi(y)=y^{2} \ln (y)$ or the Gaussian $\phi(y)=e^{-\gamma y^{2}}$ with $\gamma>0$, as proposed in 33 .

Consider $\Phi \in \mathbb{R}^{q \times q}$ the matrix defined by $\Phi_{(i, j)}:=\phi\left(\left\|X_{i}-X_{j}\right\|\right)$, for $i, j=$ $1, \ldots, q$, and $M_{P} \in \mathbb{R}^{q \times(n+1)}$ the matrix whose $i$-th line is given by [1 $\left.X_{i}^{T}\right]$. Thus, the cubic RBF model that interpolates the points $\left(X_{1}, f\left(X_{1}\right)\right), \ldots,\left(X_{q}, f\left(X_{q}\right)\right)$ is obtained by solving the system,

$$
\left(\begin{array}{cc}
\Phi & M_{P} \\
M_{P}^{T} & 0
\end{array}\right)\left(\begin{array}{l}
\eta \\
c
\end{array}\right)=\left(\begin{array}{c}
F \\
0_{n+1}
\end{array}\right)
$$

where $F=\left(f\left(X_{1}\right), \ldots, f\left(X_{q}\right)\right), \eta=\left(\eta_{1}, \ldots, \eta_{q}\right)^{T} \in \mathbb{R}^{q}$ and $c=\left(c_{1}, \ldots, c_{n+1}\right) \in$ $\mathbb{R}^{n+1}$ are the coefficients of the linear function $p$. The coefficient matrix in (27) is invertible if and only if $\operatorname{rank}\left(M_{P}\right)=n+1$, which is equivalent to having a subset of $n+1$ affinely independent points among the sample set $\mathcal{A}_{k-1}, 30,33,36$. Along the iterative process, the best points are added to the sample set to update the RBF model ensuring that the $M_{P}$ matrix will always have rank $n+1$.

\subsubsection{Best trial point selection}

The RBF model $S_{k}$ and the infeasibility measure $h$ are used to select the best trial point $X_{k}^{t^{*}}$ from the set of trial points $X_{k}^{t}$, for $t=1, \ldots, n_{t}$. The concept of non-dominance (4) is considered, replacing $\left(f^{j}, h^{j}\right)$ by $\left(S_{k}\left(X_{k}^{t}\right), h\left(X_{k}^{t}\right)\right)$. So, a point $Y$, or equivalently the pair $\left(S_{k}(Y), h(Y)\right)$, is non-dominated by the pair $\left(S_{k}\left(X_{k}^{t}\right), h\left(X_{k}^{t}\right)\right)$, if

$$
\left.S_{k}(Y)+\alpha h(Y) \leq S_{k}\left(X_{k}^{t}\right) \quad \text { or } \quad h(Y) \leq(1-\alpha) h\left(X_{k}^{t}\right)\right)
$$

Based on this, the best trial point is selected according to the following definition.

Definition 2 The best trial point $X_{k}^{t *}$ at the iteration $k$ is the trial point nondominated by any other pair $\left(S_{k}\left(X_{k}^{t}\right), h\left(X_{k}^{t}\right)\right)$, according to 28), with the smallest value of the infeasibility measure $h$.

After the selection of the best trial point $X_{k}^{t *}$, the algorithm verifies if such point is accepted by the current filter. So, the value $f\left(X_{k}^{t^{*}}\right)$ is computed. If $X_{k}^{t *} \notin$ $\overline{\mathcal{F}}_{k-1}$, it is accepted as the new iterate $X_{k}$ and it is included to the set $\mathcal{A}_{k}$ of the points used by the RBF model for the next iteration. Otherwise, the iteration is unsuccessful and we keep the best current point from the previous iteration.

\subsubsection{The algorithm}

Now we present the Filter-based stochastic algorithm FbSA assisted by Radial Basis Function surrogate models to approximate the objective function. 


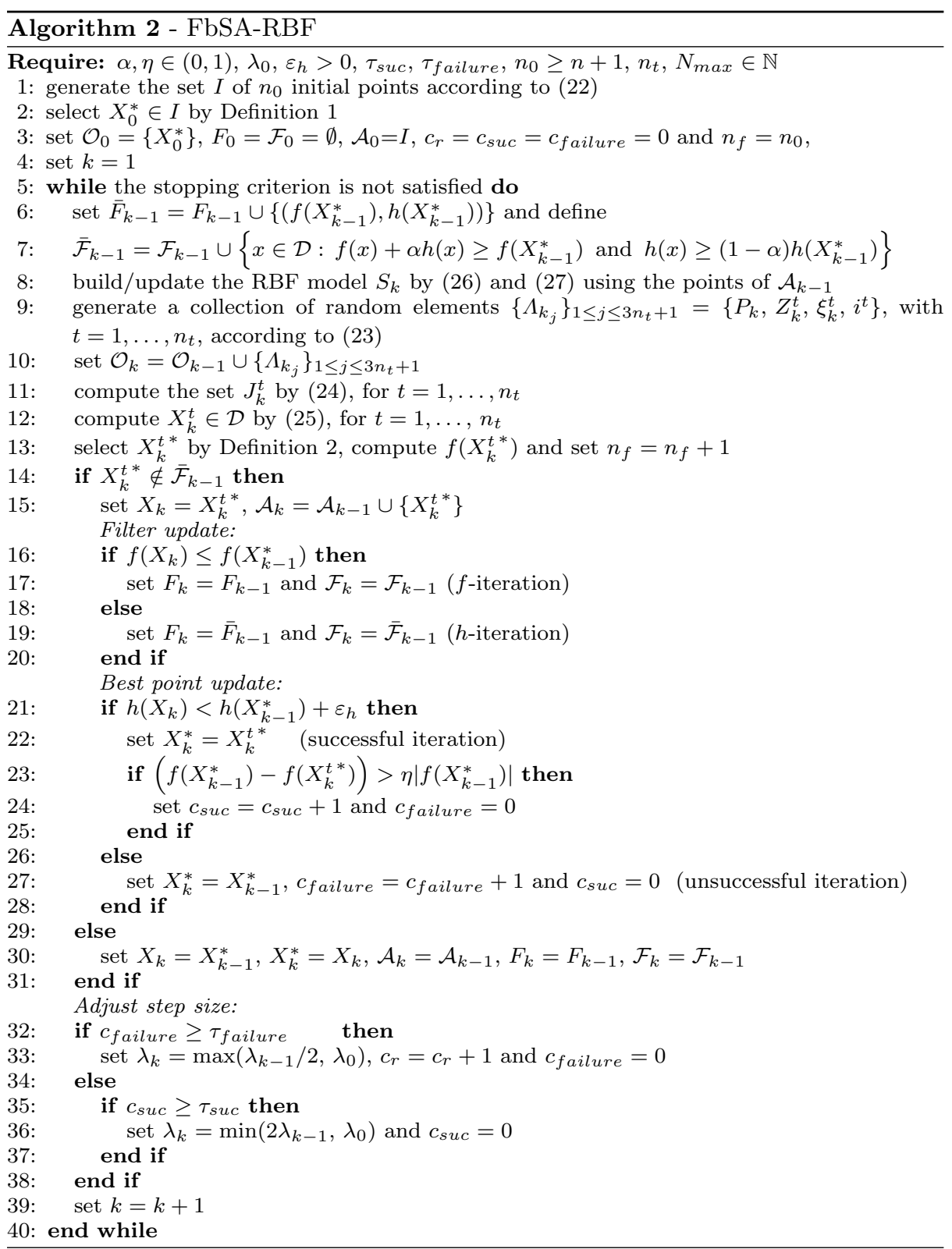

\subsection{Convergence analysis}

This section is dedicated to prove that Algorithm 2 satisfies the sufficient conditions established in Theorems 1 and 3 , ensuring its almost surely convergence.

In FbSA-RBF algorithm, the choice of the coordinates to be perturbed follows a uniform distribution, while the magnitude of perturbation follows a normal distribution. We will prove that the sequence $\left(X_{k}^{*}\right)$ generated by the algorithm admits 
a subsequence where all components have a random perturbation with normal distribution. For that, next lemma shows that the existence of such subsequence is ensured when the probability $P_{k} \in(0,1]$ is greater than a positive constant.

Lemma 3 Consider the sequence $\left(X_{k}^{*}\right)$ generated by Algorithm 2 and assume that there exists a constant $\bar{p}>0$ such that the probability $P_{k} \geq \bar{p}$, for all $k \in \mathbb{N}$. Then, there exists a subsequence $\left(X_{k_{j}}^{*}\right)$ in $\mathcal{D}$ where all components of all of its terms have random perturbations with normal distribution. More precisely,

$$
\left(X_{k_{j}}^{*}\right)_{i}=\min \left\{u_{i}, \max \left\{\left(X_{\left(k_{j}\right)-1}^{*}\right)_{i}+\left(\xi_{k_{j}}\right)_{i}, \ell_{i}\right\}\right\}
$$

with $\left(\xi_{k_{j}}\right)_{i} \sim N\left(0,\left(\lambda_{k_{j}}\right)_{i}^{2}\right)$, for $i=1, \ldots, n$.

Proof Consider the success event $E_{k}$ as the one in which all the coordinates of $X_{k}^{*}$ have a random perturbation with normal distribution. In this case, according to (24),

$$
\left[E_{k}\right]=\left[\left(Z_{k}\right)_{i} \leq P_{k}, \quad \text { for all } i=1, \ldots, n\right],
$$

where $\left(Z_{k}\right)_{i} \sim U[0,1]$. Since the events $\left[\left(Z_{k}\right)_{1} \leq P_{k}\right], \ldots,\left[\left(Z_{k}\right)_{n} \leq P_{k}\right]$ are independent, we have

$$
P\left(E_{k}\right)=\prod_{i=1}^{n} P\left(\left(Z_{k}\right)_{i} \leq P_{k}\right) .
$$

As each $\left(Z_{k}\right)_{i} \sim U[0,1]$ we have that the density function is given by

$$
g(z)=\left\{\begin{array}{l}
1, \text { if } z \in[0,1] \\
0, \text { otherwise }
\end{array}\right.
$$

Thus, for each $i$,

$$
P\left(\left(Z_{k}\right)_{i} \leq P_{k}\right)=\int_{-\infty}^{P_{k}} g(z) d z=\int_{-\infty}^{0} 0 d z+\int_{0}^{P_{k}} 1 d z=P_{k} .
$$

Substituting in (30), we have

$$
P\left(E_{k}\right)=\prod_{i=1}^{n} P_{k}=P_{k}^{n} .
$$

By the hypothesis that $P_{k} \geq \bar{p}>0$, it follows

$$
\sum_{k=1}^{\infty} P\left(E_{k}\right)=\sum_{k=1}^{\infty} P_{k}^{n} \geq \sum_{k=1}^{\infty} \bar{p}^{n}=\bar{p}^{n} \sum_{k=1}^{\infty} 1=\infty .
$$

Therefore, by Lemma of Borel-Cantelli [4],

$$
P\left(E_{k} \text { infinite times }\right)=1 .
$$

Thus, the sequence $\left(X_{k}^{*}\right)$ admits a subsequence $\left(X_{k_{j}}^{*}\right)$ such that all components of each element of the sequence are perturbed. By the algorithm, the terms of the subsequence $\left(X_{k_{j}}\right)$ are computed, coordinates to coordinates, by

$$
\left(X_{k_{j}}^{*}\right)_{i}=\min \left\{u_{i}, \max \left\{\left(X_{\left(k_{j}\right)-1}^{*}\right)_{i}+\left(\xi_{k_{j}}\right)_{i}, \ell_{i}\right\}\right\}
$$


with $\left(\xi_{k_{j}}\right)_{i} \sim N\left(0,\left(\lambda_{k_{j}}\right)_{i}^{2}\right)$, for $i=1, \ldots, n$, which completes the proof.

From last lemma, there exists a subsequence $\left(X_{k_{j}}^{*}\right)$, of the sequence $\left(X_{k}^{*}\right)$ generated by Algorithm 2, satisfying (18), which implies the convergence of the algorithm almost surely as shown in next theorem.

Theorem 4 Assume that Hypotheses H[1 - H 3 hold. Consider $\left(X_{k}^{*}\right)$ a sequence generated by Algorithm 2. Suppose that there exists $\bar{p}>0$ such that with $P_{k} \geq \bar{p}$, for all $k \in \mathbb{N}$. Then, the sequence $f\left(X_{k}^{*}\right)$ has an accumulation point that is global minimum of the $f$ over $\mathcal{D}_{V}$ almost surely. In addition, if $\mathcal{K}_{a}$ is finite, then $f\left(X_{k}^{*}\right) \rightarrow f^{*}$ almost surely. Finally, if $x^{*}$ is the unique global minimizer of $f$, then $X_{k}^{*} \rightarrow x^{*}$ almost surely.

Proof By Lemma 3 there exists a subsequence $\left(X_{k_{j}}^{*}\right)$ where all coordinates of each element have random perturbation with normal distribution, with $\left(X_{k_{j}}^{*}\right)_{i}$ given by $(29)$, where each component $\left(\xi_{k_{j}}\right)_{i} \sim N\left(0,\left(\lambda_{k_{j}}\right)_{i}^{2}\right)$ follows a normal distribution of mean 0 and standard deviation given by the square root of the eigenvalues of the covariance matrix. Thus, $\xi_{k_{j}} \sim N\left(0, V_{j}\right)$ is a random vector whose conditional distribution, given $\sigma\left(\mathcal{O}_{\left(k_{j}\right)-1}\right)$, is the multivariate normal distribution with mean 0 and covariance matrix $V_{j}$ given by

$$
V_{j}=\operatorname{Cov}\left(\xi_{k_{j}}\right)=\operatorname{diag}\left(\left(\lambda_{k_{j}}\right)_{1}^{2}, \ldots,\left(\lambda_{k_{j}}\right)_{n}^{2}\right)
$$

In this case, we have that the eigenvalues of $V_{j}$ are the variance $\left(\lambda_{k_{j}}\right)_{1}^{2}, \ldots,\left(\lambda_{k_{j}}\right)_{n}^{2}$ of the normal random perturbation added to each component of $X_{\left(k_{j}\right)-1}^{*}$. Thus, the smallest eigenvalue of $V_{j}$ is given by

$$
\bar{\lambda}_{j}:=\min _{1 \leq i \leq n}\left(\lambda_{k_{j}}\right)_{i}^{2}>0
$$

Consequently, the results follow from Theorem 3

\section{Numerical experiments}

In this section, we report the numerical experiments to illustrate the practical performance of the proposed FbSA-RBF when solving three benchmark sets of constrained global problems.

First, a comparison is carried out with other methods in the literature, which involve different approaches for handling the constraints, namely the A2-SF, P-BS AFS and EPGO, presented in [12,28, 42, using the set of problems described in 6]. Second, well-known engineering design problems presented in [43] are used in order to compare to A2-SF algorithm. In the third experiment, a set of problems described in 26,29] is used to compare the performance of FbSA-RBF with CARSRBF, CPRS-RBF and ConstrLMSRBF algorithms [30,36, which involve RBF surrogate models. 


\subsection{Implementation details}

In the two first numerical experiments, stopping conditions based on the values of $h$ and $f$ are used. Thus, the proposed FbSA-RBF algorithm stops when the best current point $X_{k}^{*}$ satisfies

$$
f\left(X_{k}^{*}\right) \leq f^{*}+\varepsilon_{f} \quad \text { and } \quad h\left(X_{k}^{*}\right) \leq \varepsilon_{h}
$$

where $\varepsilon_{f}$ is the accuracy error required in the value of the objective function, $\varepsilon_{h}$ is the tolerance of the constraint violation and $f^{*}$ is the best-known solution available in the literature. Besides, if (31) does not hold, the FbSA-RBF algorithm stops if it reaches the maximum number of function evaluations $N_{\max }=1000$ allowed. The accuracy required for the objective function and constraint violation were the same as those used in [28, 42,,$\varepsilon_{f}=10^{-4}$ and $\varepsilon_{h}=10^{-8}$, respectively.

The probability of perturbing the coordinates $P_{k}$ is computed by

$$
P_{k}=\left(1-\frac{\log \left(n_{f}-n_{0}+1\right)}{\log \left(N_{\max }-n_{0}\right)}\right) \min \left\{\frac{20}{n}, 1\right\}
$$

where $n$ is the dimension of the problem, $n_{0}=2(n+1)$ is the number of initial points, $n_{f}$ is the number of function evaluations until the iteration $k$. As the algorithm evaluates the objective function once per iteration, as $k$ increases, the probability tends to decrease. This is reasonable since at the end of the iterative process it is expected that the best current point is close to the solution of the problem and therefore major perturbations are not need.

The number of trial points $n_{t}$ generated at each iteration varies according to the dimension of the problem. The values used in the numerical experiments of FbSA-RBF were $n_{t}=\min \{100 n, 5000\}$, the maximum tolerance of failures,

$\tau_{\text {failure }}=\max \{5, n\}$, and the maximum tolerance of success, $\tau_{\text {suc }}=3$, as suggested in 39 .

Since the algorithm relies on some random parameters and variables, each problem was solved 30 times. Problems with equality constraints were reformulated to inequality constrained problems, by considering $\left|c_{i}(x)\right| \leq 10^{-4}$, for $i \in \mathcal{E}$. The tests were performed in a notebook ASUSTek Intel Core i7-6700HQ, CPU 2.60GHz, with 16GB RAM, 64-bit, using Matlab R2017a.

\subsection{Numerical results}

In the first experiment, a set of 20 global optimization test problems with general constraints described in 6 ] is used to illustrate the practical performance of FbSARBF. The number of variables ranges from 2 to 10 and the number of constraints ranges from 1 to 12. The results of this study are compared to A2-SF, P-BS AFS and EPGO.

The A2-SF [28] combines the filter methodology, with a slanting filter rule, and the DDS method. The P-BS AFS [42] is a population-based filter framework, where the subproblems are globally solved by an artificial fish swarm stochastic algorithm. The EPGO [12, uses an exact penalty approach based on the DIRECT to globally solve the subproblems. 
Table 1 presents the numerical results of the FbSA-RBF, A2-SF, P-BF AFS and EPGO for this set of problems. The first three columns display the data of the problem: the identification $(\mathrm{P})$; the dimension $(n)$; the global optimum known $\left(f^{*}\right)$. The next columns display, for each algorithm, the results obtained among the 30 runs: the best solution obtained $\left(f_{\text {best }}\right)$, the median $\left(f_{m e d}\right)$, the infeasibility measure at the best point $\left(h_{\text {best }}\right)$, the median of infeasibility measure $\left(h_{\text {med }}\right)$ and the average number of function evaluations $\left(n_{f_{\text {avg }}}\right)$. The numerical results of the A2-SF, P-BF AFS and EPGO were taken from [28], [42] and [12], respectively.

Table 1: Numerical results for problems described in 6]

\begin{tabular}{|c|c|c|c|c|c|c|c|c|}
\hline $\mathrm{P}$ & $n$ & $f^{*}$ & Algorithm & $f_{\text {best }}$ & $f_{\text {med }}$ & $h_{\text {best }}$ & $h_{m e d}$ & $n_{f_{a v g}}$ \\
\hline \multirow[t]{4}{*}{1} & \multirow[t]{4}{*}{5} & \multirow[t]{4}{*}{$2.9313 \mathrm{e}-02$} & FbSA-RBF & 0.2724 & 141.6525 & $0.00 \mathrm{e} 00$ & $0.00 \mathrm{e} 00$ & 1000 \\
\hline & & & A2-SF & 0.1978 & 237.3387 & $1.43 \mathrm{e}-04$ & $6.49 \mathrm{e}-05$ & 9479 \\
\hline & & & P-BF AFS & 0.0956 & 1.4665 & $7.84 \mathrm{e}-07$ & $*$ & 6945 \\
\hline & & & EPGO & 0.0625 & & $2.35 \mathrm{e}-07$ & & 39575 \\
\hline \multirow[t]{4}{*}{$2 a$} & \multirow[t]{4}{*}{9} & \multirow[t]{4}{*}{$-4.0000 \mathrm{e} 02$} & FbSA-RBF & -388.622 & 0.000 & $0.00 \mathrm{e} 00$ & $0.00 \mathrm{e} 00$ & 1000 \\
\hline & & & A2-SF & -395.875 & -312.487 & $0.00 \mathrm{e} 00$ & $0.00 \mathrm{e} 00$ & 14994 \\
\hline & & & P-BF AFS & -358.650 & -308.664 & $0.00 \mathrm{e} 00$ & * & 7068 \\
\hline & & & EPGO & -134.113 & & $8.43 \mathrm{e}-04$ & & 115107 \\
\hline \multirow[t]{4}{*}{$2 \mathrm{~b}$} & \multirow[t]{4}{*}{9} & \multirow[t]{4}{*}{$-6.0000 \mathrm{e} 02$} & FbSA-RBF & -284.276 & 986.439 & $0.00 \mathrm{e} 00$ & $0.00 \mathrm{e} 00$ & 1000 \\
\hline & & & $\mathrm{A} 2-\mathrm{SF}$ & -384.423 & -301.721 & $0.00 \mathrm{e} 00$ & $0.00 \mathrm{e} 00$ & 14743 \\
\hline & & & P-BF AFS & -378.317 & -274.472 & $0.00 \mathrm{e} 00$ & & 6963 \\
\hline & & & EPGO & -768.457 & & $5.30 \mathrm{e}-04$ & & 120057 \\
\hline \multirow[t]{4}{*}{$2 \mathrm{c}$} & \multirow[t]{4}{*}{9} & \multirow[t]{4}{*}{$-7.5000 \mathrm{e} 02$} & FbSA-RBF & -684.722 & -544.883 & $0.00 \mathrm{e} 00$ & $0.00 \mathrm{e} 00$ & 1000 \\
\hline & & & $\mathrm{A} 2-\mathrm{SF}$ & -747.021 & -702.915 & $0.00 \mathrm{e} 00$ & $0.00 \mathrm{e} 00$ & 14630 \\
\hline & & & P-BF AFS & -697.452 & -657.349 & $0.00 \mathrm{e} 00$ & $*$ & 7189 \\
\hline & & & EPGO & -82.977 & & $8.43 \mathrm{e}-04$ & & 102015 \\
\hline \multirow[t]{4}{*}{$2 \mathrm{~d}$} & \multirow[t]{4}{*}{10} & \multirow{4}{*}{$-4.0000 \mathrm{e} 02$} & FbSA-RBF & -400.000 & -50.386 & $0.00 \mathrm{e} 00$ & $0.00 \mathrm{e} 00$ & 1000 \\
\hline & & & $\mathrm{A} 2-\mathrm{SF}$ & -399.900 & -347.957 & $0.00 \mathrm{e} 00$ & $0.00 \mathrm{e} 00$ & 14286 \\
\hline & & & P-BF AFS & -399.118 & -394.563 & $0.00 \mathrm{e} 00$ & & 6526 \\
\hline & & & EPGO & -385.170 & & $0.00 \mathrm{e} 00$ & & 229773 \\
\hline \multirow[t]{4}{*}{$3 a$} & \multirow[t]{4}{*}{6} & \multirow[t]{4}{*}{$-3.8880 \mathrm{e}-01$} & FbSA-RBF & -0.3889 & -0.3795 & $0.00 \mathrm{e} 00$ & $0.00 \mathrm{e} 00$ & 1000 \\
\hline & & & A2-SF & -0.3878 & -0.3747 & $0.00 \mathrm{e} 00$ & $0.00 \mathrm{e} 00$ & 15858 \\
\hline & & & P-BF AFS & -0.3888 & -0.3842 & $5.22 \mathrm{e}-04$ & $*$ & 7495 \\
\hline & & & EPGO & -0.3861 & & $1.02 \mathrm{e}-06$ & & 48647 \\
\hline \multirow[t]{4}{*}{$3 \mathrm{~b}$} & \multirow[t]{4}{*}{2} & \multirow[t]{4}{*}{$-3.8881 \mathrm{e}-01$} & FbSA-RBF & -0.3888 & -0.3885 & $0.00 \mathrm{e} 00$ & $0.00 \mathrm{e} 00$ & 907 \\
\hline & & & A2-SF & -0.3888 & -0.3883 & $0.00 \mathrm{e} 00$ & $0.00 \mathrm{e} 00$ & 6512 \\
\hline & & & P-BF AFS & -0.3888 & -0.3888 & $0.00 \mathrm{e} 00$ & * & 1041 \\
\hline & & & EPGO & -0.3888 & & $0.00 \mathrm{e} 00$ & & 3449 \\
\hline \multirow[t]{4}{*}{4} & 2 & $-6.6666 \mathrm{e} 00$ & FbSA-RBF & -6.6666 & -6.6665 & $0.00 \mathrm{e} 00$ & $0.00 \mathrm{e} 00$ & 361 \\
\hline & & & $\mathrm{A} 2-\mathrm{SF}$ & -6.6666 & -6.6662 & $0.00 \mathrm{e} 00$ & $0.00 \mathrm{e} 00$ & 5483 \\
\hline & & & P-BF AFS & -6.6667 & -6.6665 & $0.00 \mathrm{e} 00$ & $*$ & 493 \\
\hline & & & EPGO & -6.6666 & & $0.00 \mathrm{e} 00$ & & 3547 \\
\hline 5 & 3 & $2.0116 \mathrm{e} 02$ & FbSA-RBF & 201.159 & 201.159 & $0.00 \mathrm{e} 00$ & $0.00 \mathrm{e} 00$ & 1000 \\
\hline & & & $\mathrm{A} 2-\mathrm{SF}$ & 201.159 & 201.157 & $0.00 \mathrm{e} 00$ & $3.57 \mathrm{e}-02$ & 2930 \\
\hline & & & P-BF AFS & 201.159 & 201.159 & $8.11 \mathrm{e}-07$ & * & 2999 \\
\hline & & & EPGO & 201.159 & & $1.66 \mathrm{e}-04$ & & 14087 \\
\hline 6 & 2 & $3.7629 \mathrm{e} 02$ & FbSA-RBF & 376.292 & 376.293 & $0.00 \mathrm{e} 00$ & $0.00 \mathrm{e} 00$ & 1000 \\
\hline & & & A2-SF & 376.305 & 376.986 & $0.00 \mathrm{e} 00$ & $0.00 \mathrm{e} 00$ & 6079 \\
\hline & & & P-BF AFS & 376.293 & 376.304 & $0.00 \mathrm{e} 00$ & $*$ & 1335 \\
\hline & & & EPGO & 0.470 & & $2.05 \mathrm{e}-05$ & & 1523 \\
\hline 7 & 2 & $-2.8284 \mathrm{e} 00$ & FbSA-RBF & -2.8284 & -2.8283 & $0.00 \mathrm{e} 00$ & $0.00 \mathrm{e} 00$ & 194 \\
\hline & & & A2-SF & -2.8284 & -2.8230 & $0.00 \mathrm{e} 00$ & $0.00 \mathrm{e} 00$ & 4829 \\
\hline & & & P-BF AFS & -2.8284 & -2.8283 & $0.00 \mathrm{e} 00$ & $*$ & 920 \\
\hline & & & EPGO & -2.8058 & & $0.00 \mathrm{e} 00$ & & 13187 \\
\hline
\end{tabular}




\begin{tabular}{|c|c|c|c|c|c|c|c|c|}
\hline $\mathrm{P}$ & $n$ & $f^{*}$ & Algorithm & $f_{\text {best }}$ & $f_{\text {med }}$ & $h_{\text {best }}$ & $h_{m e d}$ & $n_{f_{\text {avg }}}$ \\
\hline 8 & 2 & $-1.1870 \mathrm{e} 02$ & $\begin{array}{l}\text { FbSA-RBF } \\
\text { A2-SF } \\
\text { P-BF AFS } \\
\text { EPGO }\end{array}$ & $\begin{array}{l}-118.704 \\
-118.703 \\
-118.704 \\
-118.704\end{array}$ & $\begin{array}{r}-86.422 \\
-115.138 \\
-118.698\end{array}$ & $\begin{array}{l}0.00 \mathrm{e} 00 \\
0.00 \mathrm{e} 00 \\
0.00 \mathrm{e} 00 \\
0.00 \mathrm{e} 00\end{array}$ & $\begin{array}{r}0.00 \mathrm{e} 00 \\
0.00 \mathrm{e} 00 \\
*\end{array}$ & $\begin{array}{l}1000 \\
5904 \\
1521 \\
7621\end{array}$ \\
\hline 9 & 6 & $-1.3402 \mathrm{e} 01$ & $\begin{array}{l}\text { FbSA-RBF } \\
\text { A2-SF } \\
\text { P-BF AFS } \\
\text { EPGO }\end{array}$ & $\begin{array}{l}-13.4019 \\
-13.4019 \\
-13.4018 \\
-13.4026\end{array}$ & $\begin{array}{l}-13.4019 \\
-13.3916 \\
-13.4007\end{array}$ & $\begin{array}{r}0.00 \mathrm{e} 00 \\
0.00 \mathrm{e} 00 \\
0.00 \mathrm{e} 00 \\
1.35 \mathrm{e}-04\end{array}$ & $\begin{array}{r}0.00 \mathrm{e} 00 \\
0.00 \mathrm{e} 00 \\
*\end{array}$ & $\begin{array}{r}1000 \\
8187 \\
1839 \\
68177\end{array}$ \\
\hline 10 & 2 & $7.4178 \mathrm{e}-01$ & $\begin{array}{l}\text { FbSA-RBF } \\
\text { A2-SF } \\
\text { P-BF AFS } \\
\text { EPGO }\end{array}$ & $\begin{array}{l}0.7418 \\
0.7419 \\
0.7418 \\
0.7420\end{array}$ & $\begin{array}{l}0.7418 \\
0.7436 \\
0.7418\end{array}$ & $\begin{array}{l}0.00 \mathrm{e} 00 \\
0.00 \mathrm{e} 00 \\
0.00 \mathrm{e} 00 \\
0.00 \mathrm{e} 00\end{array}$ & $\begin{array}{r}0.00 \mathrm{e} 00 \\
0.00 \mathrm{e} 00 \\
*\end{array}$ & $\begin{array}{r}68 \\
5733 \\
2126 \\
6739\end{array}$ \\
\hline 11 & 2 & $-5.0000 \mathrm{e}-01$ & $\begin{array}{l}\text { FbSA-RBF } \\
\text { A2-SF } \\
\text { P-BF AFS } \\
\text { EPGO }\end{array}$ & $\begin{array}{l}-0.5000 \\
-0.5000 \\
-0.5000 \\
-0.5000\end{array}$ & $\begin{array}{l}-0.4999 \\
-0.4982 \\
-0.5000\end{array}$ & $\begin{array}{l}0.00 \mathrm{e} 00 \\
0.00 \mathrm{e} 00 \\
0.00 \mathrm{e} 00 \\
0.00 \mathrm{e} 00\end{array}$ & $\begin{array}{r}0.00 \mathrm{e} 00 \\
0.00 \mathrm{e} 00 \\
*\end{array}$ & $\begin{array}{r}80 \\
6135 \\
782 \\
3579\end{array}$ \\
\hline 12 & 2 & $-1.6739 \mathrm{e} 01$ & $\begin{array}{l}\text { FbSA-RBF } \\
\text { A2-FF } \\
\text { P-BF AFS } \\
\text { EPGO }\end{array}$ & $\begin{array}{l}-16.7393 \\
-16.6486 \\
-16.7389 \\
-16.7389 \\
\end{array}$ & $\begin{array}{l}-16.7321 \\
-15.5805 \\
-16.7389\end{array}$ & $\begin{array}{r}0.00 \mathrm{e} 00 \\
0.00 \mathrm{e} 00 \\
0.00 \mathrm{e} 00 \\
5.36 \mathrm{e}-06 \\
\end{array}$ & $\begin{array}{r}0.00 \mathrm{e} 00 \\
3.02 \mathrm{e}-05 \\
*\end{array}$ & $\begin{array}{r}1000 \\
4159 \\
35 \\
3499 \\
\end{array}$ \\
\hline 13 & 3 & $1.8935 \mathrm{e} 02$ & $\begin{array}{l}\text { FbSA-RBF } \\
\text { A2-SF } \\
\text { P-BF AFS } \\
\text { EPGO }\end{array}$ & $\begin{array}{l}271.994 \\
278.942 \\
189.345 \\
195.955\end{array}$ & $\begin{array}{l}287.201 \\
280.580 \\
253.937\end{array}$ & $\begin{array}{r}0.00 \mathrm{e} 00 \\
1.45 \mathrm{e}-05 \\
0.00 \mathrm{e} 00 \\
9.21 \mathrm{e}-04\end{array}$ & $\begin{array}{r}1.17 \mathrm{e}-04 \\
3.30 \mathrm{e}-01 \\
*\end{array}$ & $\begin{array}{l}1000 \\
4601 \\
4031 \\
8085\end{array}$ \\
\hline 14 & 4 & $-4.5142 \mathrm{e} 00$ & $\begin{array}{l}\text { FbSA-RBF } \\
\text { A2-SF } \\
\text { P-BF AFS } \\
\text { EPGO }\end{array}$ & $\begin{array}{l}-4.5142 \\
-4.5142 \\
-4.5142 \\
-4.3460\end{array}$ & $\begin{array}{l}-4.5142 \\
-4.4808 \\
-4.5139\end{array}$ & $\begin{array}{r}0.00 \mathrm{e} 00 \\
0.00 \mathrm{e} 00 \\
0.00 \mathrm{e} 00 \\
9.22 \mathrm{e}-05\end{array}$ & $\begin{array}{r}0.00 \mathrm{e} 00 \\
0.00 \mathrm{e} 00 \\
*\end{array}$ & $\begin{array}{r}523 \\
8520 \\
2028 \\
19685\end{array}$ \\
\hline 15 & 3 & $0.0000 \mathrm{e} 00$ & $\begin{array}{l}\text { FbSA-RBF } \\
\text { A2-SF } \\
\text { P-BF AFS } \\
\text { EPGO }\end{array}$ & $\begin{array}{l}0.0000 \\
0.0000 \\
0.0000 \\
0.0000\end{array}$ & $\begin{array}{l}0.0000 \\
0.0000 \\
0.0000\end{array}$ & $\begin{array}{r}0.00 \mathrm{e} 00 \\
2.03 \mathrm{e}-05 \\
9.11 \mathrm{e}-07 \\
4.94 \mathrm{e}-05\end{array}$ & $\begin{array}{r}1.25 \mathrm{e}-02 \\
1.91 \mathrm{e}-02 \\
*\end{array}$ & $\begin{array}{l}1000 \\
4729 \\
3593 \\
1645\end{array}$ \\
\hline 16 & 5 & $7.0492 \mathrm{e}-01$ & $\begin{array}{l}\text { FbSA-RBF } \\
\text { A2-SF } \\
\text { P-BF AFS } \\
\text { EPGO }\end{array}$ & $\begin{array}{l}0.7050 \\
0.7049 \\
0.7049 \\
0.7181\end{array}$ & $\begin{array}{l}0.7050 \\
0.7050 \\
0.7049\end{array}$ & $\begin{array}{r}0.00 \mathrm{e} 00 \\
0.00 \mathrm{e} 00 \\
0.00 \mathrm{e} 00 \\
2.00 \mathrm{e}-04\end{array}$ & $\begin{array}{r}0.00 \mathrm{e} 00 \\
0.00 \mathrm{e} 00 \\
*\end{array}$ & $\begin{array}{r}21 \\
121 \\
447 \\
22593\end{array}$ \\
\hline
\end{tabular}

*information not available at source

From Table 1] we can conclude that the proposed algorithm presents competitive results when compared with the P-BF AFS, A2-SF and EPGO algorithms. Using less function evaluations, FbSA-RBF reaches the global optimum value known $f^{*}$ on 15 out of 20 problems. Furthermore, the median objective function values are close to $f^{*}$ for 12 of them. Four of the problems (1, 2a, 2b and 2c) are not solved by any algorithm, although some of them obtained feasible solutions.

The second experiment involves nine more complex and real application engineering design problems described in [43], since problems of practical interest are important for assessing the effectiveness of the proposed algorithm. In this set of problems, the number of design variables ranges from 2 to 8 and the number of inequality constraints ranges from 1 to 11 .

Table 2 presents the results obtained by FbSA-RBF and A2-SF [28. The columns related to the infeasibility measures are omitted since both algorithms 
produced feasible solutions in all runs. Using less function evaluations, FbSA$\mathrm{RBF}$ reaches best solutions in all problems, comparing to A2-SF. None algorithm solved with a good accuracy the problems C. Vessel, Heat E. and W. Beam.

Table 2 Numerical results for engineering design problems described in 43

\begin{tabular}{|c|c|c|c|c|c|c|}
\hline $\mathrm{P}$ & $n$ & $f^{*}$ & Algorithm & $f_{\text {best }}$ & $f_{m e d}$ & $n_{f_{\text {avg }}}$ \\
\hline \multirow[t]{2}{*}{ C.Vessel } & 5 & 5868.7650 & FbSA-RBF & 5885.4030 & 6263.9640 & 1000 \\
\hline & & & A2-SF & 5898.3626 & 6327.0383 & 10966 \\
\hline \multirow[t]{2}{*}{ D.Brake } & 4 & 0.1274 & FbSA-RBF & 0.1274 & 0.1274 & 793 \\
\hline & & & A2-SF & 0.1274 & 0.1283 & 6770 \\
\hline \multirow[t]{2}{*}{ F.B.Truss } & 4 & 1400.000 & FbSA-RBF & 1400.0000 & 1400.0000 & 14 \\
\hline & & & A2-SF & 1400.0000 & 1400.0000 & 336 \\
\hline \multirow[t]{2}{*}{ Heat E. } & 8 & 7049.2480 & FbSA-RBF & 7051.1860 & 7666.1930 & 1000 \\
\hline & & & A2-SF & 7075.0293 & 8340.3915 & 17826 \\
\hline \multirow[t]{2}{*}{ S.Reducer } & 7 & 2994.4991 & FbSA-RBF & 2994.4840 & 3089.5885 & 1000 \\
\hline & & & A2-SF & 2994.4840 & 2994.5185 & 15146 \\
\hline \multirow[t]{2}{*}{ T.Column } & 2 & 26.5313 & FbSA-RBF & 26.5314 & 27.0672 & 1000 \\
\hline & & & A2-SF & 26.5342 & 26.6429 & 5884 \\
\hline \multirow[t]{2}{*}{ T.Spring } & 3 & 0.0127 & FbSA-RBF & 0.0127 & 0.0129 & 1000 \\
\hline & & & A2-SF & 0.0127 & 0.0140 & 7598 \\
\hline \multirow{2}{*}{ T.B.Truss } & 2 & 263.8958 & FbSA-RBF & 263.8959 & 263.8964 & 819 \\
\hline & & & A2-SF & 263.9086 & 264.0120 & 6649 \\
\hline \multirow[t]{2}{*}{ W.Beam } & 4 & 2.3809 & FbSA-RBF & 2.4568 & 3.2971 & 1000 \\
\hline & & & A2-SF & 2.5942 & 5.2176 & 11016 \\
\hline
\end{tabular}

The third set of experiments aims to compare the results produced by FbSARBF and other methods that use RBF surrogate models, the CARS-RBF, the Constrained Accelerated Random Search algorithm; CPRS-RBF, the RBF-assisted version of the Constrained Pure Random Search and ConstrLMSRBF, an extension of the Local Metric Stochastic RBF reported in 30. The CARS-RBF and ConstrLMSRBF use surrogates to approximate the objective and constraint functions.

A set of 26 test problems whose dimensions range from 2 to 20 and the number of inequality constraints ranges from 1 to 38 is considered. Fifteen of them belong to the G's collection, a set of well-known constrained optimization test problems developed for the "CEC 2006 Competition on Constrained Real-Parameter Optimization" and described in 26. The remaining problems are from the suit of scalable functions developed for the "CEC 2010 Competition on Constrained Real-Parameter Optimization", fully described in [29], using the 10-dimensional instances of them. The problems labeled MOD are obtained from the corresponding original version by relaxing the equality constraints. The description of these 26 test problems can be found in [30.

For a fair comparison, the algorithms stop when the maximum number of objective function evaluations $N_{\max }=100(n+1)$ is attained, as suggested in [30]. The results are summarized in Table 3 , where $f_{a v g}$ and stdv represent, respectively, the mean and the standard deviation of the best objective function values among of the feasible solutions of the 30 runs. In addition, to the FbSA-RBF algorithm, we included the column (succ) with the percentage of runs that produced feasible 
solutions (successful runs) among the 30 runs. This information is not available for the other methods in comparison.

FbSA-RB is the algorithm with best $f_{\text {avg }}$ results on 15 out of the 26 problems. For example, FbSA-RBF presented much better results than the other algorithms for problems G01, G08, G09, with $f_{\text {avg }}$ closer to the global optimal known. It should be noted that for the problem G09, FbSA-RBF has a success rate of $93 \%$, which means that feasible solutions have been obtained in 28 of the 30 runs.

To complement the information of the table, Figure 2 presents the performance profile, as proposed in [13, for the four algorithms for solving this set of 26 problems, related to the performance measure $\left|f_{a v g}-f^{*}\right| / \max \left(1,\left|f^{*}\right|\right)$ as suggested in [11. The plot reports (on the vertical axis) the percentage of problems solved by each algorithm that is within a certain threshold, $t$, (on the horizontal axis) of the best result. The higher the percentage the better. A higher value for $t=1$ means that the corresponding algorithm achieves the smallest value of the metric mostly. 
Table 3 Comparison between FbSA-RBF and other methods using RBF surrogate models

\begin{tabular}{|c|c|c|c|c|c|c|c|c|c|c|c|}
\hline \multirow[b]{2}{*}{ Problem } & \multirow[b]{2}{*}{$f^{*}$} & \multicolumn{4}{|c|}{ FbSA-RBF } & \multicolumn{2}{|c|}{ CARS-RBF } & \multicolumn{2}{|c|}{ CPRS-RBF } & \multicolumn{2}{|c|}{ ConstrLMSRBF } \\
\hline & & $f_{\text {best }}$ & $f_{\text {avg }}$ & stdv & succ & $f_{\text {avg }}$ & stdv & $f_{\text {avg }}$ & stdv & $f_{\text {avg }}$ & stdv \\
\hline G01 & $-1.5000 \mathrm{e}+01$ & -15.000000 & $-1.4949 e+01$ & $1.0688 \mathrm{e}-01$ & 100 & $.1982 \mathrm{e}+00$ & $5685 \mathrm{e}-01$ & $.7125 \mathrm{e}+00$ & $1.4856 \mathrm{e}-01$ & $-1.2358 \mathrm{e}+01$ & $3.1299 \mathrm{e}-01$ \\
\hline G02 & $-8.0362 \mathrm{e}-01$ & -0.739492 & $-5.3021 \mathrm{e}-01$ & & 100 & $-5.4267 \mathrm{e}-01$ & & $-4.6623 \mathrm{e}-01$ & $5.1095 \mathrm{e}-03$ & $-3.3069 \mathrm{e}-01$ & $1.7471 \mathrm{e}-02$ \\
\hline G03N & $-1.0005 e+00$ & -0.995700 & $-7.8827 \mathrm{e}-01$ & $3.3974 \mathrm{e}-01$ & 100 & $-6.9302 \mathrm{e}-01$ & $3.7562 \mathrm{e}-06$ & $-2.1476 \mathrm{e}-05$ & $1.1090 \mathrm{e}-05$ & $-6.4612 \mathrm{e}-01$ & $3.2067 \mathrm{e}-02$ \\
\hline G04 & $-3.0666 \mathrm{e}+04$ & -30665.430000 & $-3.0514 \mathrm{e}+04$ & $9.5866 \mathrm{e}+01$ & 100 & $-3.0664 \mathrm{e}+04$ & $1.0583 \mathrm{e}-01$ & $-3.0512 \mathrm{e}+04$ & $6.5526 \mathrm{e}+00$ & $-3.0664 \mathrm{e}+04$ & $1.0408 \mathrm{e}-01$ \\
\hline G05MOD & $5.1265 \mathrm{e}+03$ & 5125.470000 & $5.2668 \mathrm{e}+03$ & $2.1450 \mathrm{e}+02$ & 47 & $5.1307 \mathrm{e}+03$ & $6.8687 \mathrm{e}-01$ & $5.2860 \mathrm{e}+03$ & $1.1029 \mathrm{e}+01$ & $5.1303 \mathrm{e}+03$ & $5.3444 \mathrm{e}-01$ \\
\hline G06 & $-6.9618 \mathrm{e}+03$ & 00 & $-6.8917 e+03$ & $2.3192 \mathrm{e}+01$ & 100 & $.8478 \mathrm{e}+03$ & 1.0763 & $-6.1040 \mathrm{e}+03$ & +01 & $-6.8843 e+03$ & 1.0 \\
\hline G07 & $2.4306 \mathrm{e}+01$ & 24.331230 & $2.4487 \mathrm{e}+01$ & $1.0720 \mathrm{e}-01$ & 100 & $2.4647 \mathrm{e}+01$ & $1.9148 \mathrm{e}-02$ & $5.0061 \mathrm{e}+02$ & $4.7844 \mathrm{e}+01$ & $2.4560 \mathrm{e}+01$ & $1.2624 \mathrm{e}-02$ \\
\hline G08 & $-9.5825 \mathrm{e}-02$ & -0.095825 & $-9.5822 \mathrm{e}-02$ & $9.9968 \mathrm{e}-06$ & 100 & $-6.2234 \mathrm{e}-02$ & & $-8.6539 \mathrm{e}-02$ & & & \\
\hline G09 & $6.8063 \mathrm{e}+02$ & 1500 & $6.8091 \mathrm{e}+02$ & $1.3249 \mathrm{e}-01$ & 93 & 7.788 & $1.8201 \mathrm{e}+01$ & $9.7861 \mathrm{e}+02$ & $2.3571 \mathrm{e}+01$ & $9.9629 \mathrm{e}+02$ & +01 \\
\hline G10 & $7.0492 \mathrm{e}+03$ & 7073.403000 & $7.3638 \mathrm{e}+03$ & $1.5763 \mathrm{e}+02$ & 100 & $7.6393 \mathrm{e}+03$ & $9.1632 \mathrm{e}+01$ & $1.3513 \mathrm{e}+04$ & $2.9508 \mathrm{e}+02$ & $7.2959 \mathrm{e}+03$ & $2.9906 \mathrm{e}+01$ \\
\hline G13I & $5.3942 \mathrm{e}-02$ & 0.123741 & $7.4965 \mathrm{e}-01$ & $2.4279 \mathrm{e}-01$ & 100 & 3.576 & e- -05 & $5.2518 \mathrm{e}-03$ & $4 e-04$ & $1.3880 \mathrm{e}-02$ & -03 \\
\hline G16 & $-1.9052 \mathrm{e}+00$ & & $-1.9047 \mathrm{e}+00$ & $2.6813 \mathrm{e}-04$ & 100 & $-1.9032 \mathrm{e}$ & & $-1.7177 \mathrm{e}$ & 03 & -1.90 & \\
\hline G18 & $-8.6603 \mathrm{e}-01$ & -0.86 & $-8.5837 \mathrm{e}-01$ & $7.1484 \mathrm{e}-03$ & 100 & $-8.1354 \mathrm{e}-01$ & $1.4906 \mathrm{e}-02$ & $-2.3243 \mathrm{e}-01$ & $1.7915 \mathrm{e}-02$ & $-8.2248 \mathrm{e}-01$ & $1.3001 \mathrm{e}-02$ \\
\hline G19 & $3.2656 \mathrm{e}+01$ & 36.690370 & $5.4316 \mathrm{e}+01$ & $1.4838 \mathrm{e}+01$ & 100 & $7.7821 \mathrm{e}+01$ & $2.3259 \mathrm{e}+00$ & $7.9816 \mathrm{e}+02$ & $2.3129 \mathrm{e}+01$ & $6.4197 \mathrm{e}+01$ & $2.1127 \mathrm{e}+00$ \\
\hline G24 & $-5.5080 \mathrm{e}+00$ & & $-5.5079 \mathrm{e}+00$ & $1.3170 \mathrm{e}-04$ & 100 & $-5.5061 \mathrm{e}+00$ & & $-5.4979 \mathrm{e}+00$ & $9.8807 \mathrm{e}-04$ & $-5.3249 \mathrm{e}+00$ & \\
\hline $\mathrm{C} 01$ & $-7.4731 \mathrm{e}-01$ & -0.737633 & $-5.2942 \mathrm{e}-01$ & $9.3865 \mathrm{e}-02$ & 100 & $-4.5715 \mathrm{e}-01$ & $1.3609 \mathrm{e}-02$ & $-4.1669 \mathrm{e}-01$ & $8.7688 \mathrm{e}-03$ & $-2.9030 \mathrm{e}-01$ & $1.0710 \mathrm{e}-02$ \\
\hline & $-2.2777 \mathrm{e}+00$ & -2.191 & $-1.2200 \mathrm{e}+00$ & $5.1038 \mathrm{e}-01$ & 100 & $-2.2114 \mathrm{e}+00$ & $3.1975 \mathrm{e}-02$ & $-1.0889 e+00$ & $3.3414 \mathrm{e}-02$ & $-2.0195 \mathrm{e}+00$ & $6.6629 \mathrm{e}-02$ \\
\hline & $-4.8361 \mathrm{e}+02$ & 187.537000 & $4.6127 \mathrm{e}+02$ & $1.2256 \mathrm{e}+02$ & 83 & $-4.7165 e+02$ & $4.0030 \mathrm{e}+00$ & $-2.9240 \mathrm{e}+02$ & $6.5636 \mathrm{e}+00$ & $-4.0531 \mathrm{e}+02$ & $2.1516 \mathrm{e}+01$ \\
\hline $\mathrm{C} 06 \mathrm{~N}$ & $-5.7866 e+02$ & 379.907600 & $5.4645 \mathrm{e}+02$ & $7.7265 \mathrm{e}+01$ & 93 & $-3.6983 \mathrm{e}$ & 1.74 & $-1.5177 \mathrm{e}+02$ & $3.7059 \mathrm{e}+00$ & -3.297 & 2.22 \\
\hline $\mathrm{C} 07$ & $00000 e+00$ & & 1.160 & $1.6964 \mathrm{e}+02$ & 100 & 36011 & & $8.1804 \mathrm{e}+08$ & $1.3970 \mathrm{e}+08$ & $1.059 \mathrm{c}$ & $e+04$ \\
\hline $\mathrm{C} 08$ & $0.0000 \mathrm{e}+00$ & $1.2497 \mathrm{e}+01$ & $1.6417 \mathrm{e}+02$ & $1.5569 \mathrm{e}+02$ & 100 & $5.7753 \mathrm{e}+05$ & $1.5980 \mathrm{e}+05$ & $8.8208 \mathrm{e}+08$ & $1.4292 \mathrm{e}+08$ & $2.0133 \mathrm{e}+06$ & $3.0348 \mathrm{e}+05$ \\
\hline & $0.0000 \mathrm{e}+00$ & & $1.7233 \mathrm{e}+13$ & $7.0639 \mathrm{e}+12$ & 100 & & & $6.2069 \mathrm{e}+10$ & $9.4480 \mathrm{e}+09$ & $1.7092 \mathrm{e}+08$ & \\
\hline C10MOD & $0.0000 \mathrm{e}+00$ & $3.0016 \mathrm{e}+12$ & $1.6665 \mathrm{e}+13$ & $6.5694 \mathrm{e}+12$ & 10 & $3.5231 \mathrm{\epsilon}$ & $5.3131 \mathrm{e}+06$ & $5.1332 \mathrm{e}+10$ & $8.1646 \mathrm{e}+09$ & $1.6627 \mathrm{e}+08$ & $1.3735 \mathrm{e}+07$ \\
\hline C14 & $0.0000 \mathrm{e}+00$ & 105.564200 & $3.5730 \mathrm{e}+03$ & $4.0722 \mathrm{e}+03$ & 100 & & & & & & $2.2457 \mathrm{e}+08$ \\
\hline $\mathrm{C} 15$ & $0.0000 \mathrm{e}+00$ & & $1.2037 \mathrm{e}+$ & $2.3835 \mathrm{e}+13$ & 10 & & & $1.5982 \mathrm{e}+$ & $1.4021 \mathrm{e}+13$ & $1.6565 \mathrm{e}+$ & $1.4788 \mathrm{e}+13$ \\
\hline C17MOD & $0.0000 \mathrm{e}+00$ & 114.687300 & $5.0187 \mathrm{e}+02$ & $1.7574 \mathrm{e}+02$ & 100 & $4.8892 \mathrm{e}+00$ & $5.6902 \mathrm{e}-01$ & $3.6618 \mathrm{e}+01$ & $2.4068 \mathrm{e}+00$ & $5.7031 \mathrm{e}+00$ & $7.7777 \mathrm{e}-01$ \\
\hline
\end{tabular}


So, in terms of efficiency we can say that FbSA-RBF, CARS-RBF and ConstrLMSRBF solved about $58 \%, 31 \%$ and $15 \%$ of the problems, respectively, with the smallest value of the metric considered. On the other hand, CPRS-RBF does not solve any problem with the best performance measure. FbSA-RBF and ConstrLMSRBF are the most robust algorithm, solving all problems using no more than $4 \times 10^{5}$ and $8 \times 10^{5}$, respectively, the performance measure used by the best algorithm. Consequently, FbSA-RBF has competitive results when comparing with other strategies using RBF surrogate models.
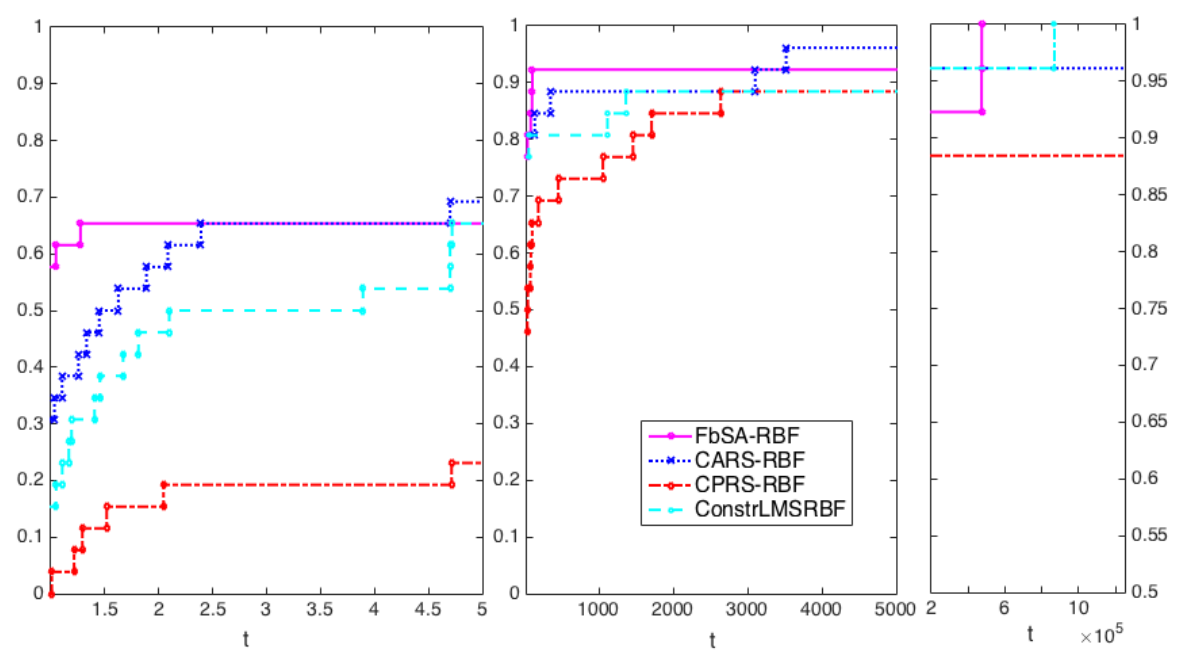

Fig. 2 Performance profile for FbSA-RBF, CARS-RBF, CPRS-RBF and ConstrLMSRBF with measure $\left|f_{\text {avg }}-f^{*}\right| / \max \left(1,\left|f^{*}\right|\right)$

\section{Conclusions}

In this paper, the general FbSA to solve nonlinear and nonconvex constrained global optimization problems is proposed. The convergence of the algorithm is almost surely guaranteed once the probability distribution used in the computation of the iterates satisfies some hypotheses. To optimize problems with computationally expensive black-box objective functions, we developed the FbSA-RBF based on the structure of the general algorithm and assisted by radial basis function surrogate models to approximate the objective function. In each iteration of the FbSA-RBF, several trial points are computed by adding random perturbations with normal distribution in the dynamically chosen coordinates of the best current point. The best of them is chosen by using a non-dominance criterion based on the values of the surrogate model and the infeasibility measure at the trial points. This point replaces the current best point if it is accepted by the filter and does not increase the infeasibility measure by more than a small positive quantity $\varepsilon_{h}$, 
from the current best one. Theoretical results concerning the sufficient conditions for the almost surely convergence of the algorithm are proved. A performance comparison of the FbSA-RBF with other algorithms in the literature, when solving 55 problems of three different sets, is presented. The numerical experiments evidenced significant savings in the number of objective function evaluations by the FbSA-RBF, providing competitive results when compared to other algorithms.

Acknowledgments.

The authors are grateful to the anonymous referees for their fruitful comments and suggestions. The first and second authors were partially supported by Brazilian Funds through CAPES and CNPq by Grants PDSE 99999.009400/2014-01 and 309303/2017-6. The research of the third and fourth authors were partially financed by Portuguese Funds through FCT (Fundação para Ciência e Tecnologia) within the Projects UIDB/00013/2020 and UIDP/00013/2020 of CMAT-UM and UIDB/00319/2020.

\section{References}

1. A. H. Aguirre, S. B. Rionda, C. A. Coello Coello, G. L. Lizrraga, and E. M. Montes. Handling constraints using multiobjective optimization concepts. Int. J. Numer. Meth. Eng., 59(15):1989-2017, 2004.

2. M. M. Ali and M. Golalikhani. An electromagnetism-like method for nonlinearly constrained global optimization. Comput. Math. Appl., 60(8):2279-2285, 2010.

3. M. M. Ali and W. X. Zhu. A penalty function-based differential evolution algorithm for constrained global optimization. Comput. Optim. Appl., 54(1):707-739, 2013.

4. C. Audet and J. E. Dennis Jr. A pattern search filter method for nonlinear programming without derivatives. SIAM J. Optimiz., 14(4):980-1010, 2004

5. H. J. C. Barbosa and A. C. C. Lemonge. An adaptive penalty method for genetic algorithms in constrained optimization problems. In H. Iba, editor, Frontiers in Evolutionary Robotics, chapter 2. IntechOpen, Rijeka, 2008.

6. E. G. Birgin, C. A. Floudas, and J. M. Martínez. Global minimization using an augmented Lagrangian method with variable lower-level constraints. Math. Program., 125:139-162, 2010.

7. A. J. Booker, J. E. Dennis, P. D. Frank, D. B. Serafini, V. Torczon, and M. W. Trosset. A rigorous framework for optimization of expensive functions by surrogates. Struct. Multidisc. Optim., 17:1-19, 1999.

8. C. M. Chin and R. Fletcher. On the global convergence of an SLP-filter algorithm that takes EQP steps. Math. Program., 96(1):161-177, 2003.

9. C. A. C. Coello. Theoretical and numerical constraint-handling techniques used with evolutionary algorithms: a survey of the state of the art. Comput. Method. Appl. M., 191(11):1245 - 1287, 2002.

10. M. F. P. Costa, F. P. Fernandes, and A. M. A. C. Rocha. Multiple solutions of mixed variable optimization by multistart Hooke and Jeeves filter method. Appl. Math. Sci., 8:2163-2179, 2014.

11. M. F. P. Costa, A. M. A. C. Rocha, and E. M. G. P. Fernandes. Filter-based direct method for constrained global optimization. J. Global Optim., 71(3):517-536, 2018.

12. G. Di Pillo, S. Lucidi, and F. Rinaldi. An approach to constrained global optimization based on exact penalty functions. J. Global Optim., 54:251-260, 2012.

13. E. D. Dolan and J. Moré. Benchmarking optimization software with performance profiles. Math. Program., 91(2):201-213, 2002.

14. N. Echebest, M. L. Shuverdt, and R. P. Vignau. An inexact restoration derivative-free filter method for nonlinear programming. Comput. Appl. Math., 2015. DOI 10.1007/s40314015-0253-0.

15. P. S. Ferreira, E. W. Karas, M. Sachine, and F. N. Sobral. Global convergence of a derivative-free inexact restoration filter algorithm for nonlinear programming. Optimization, 66:271-292, 2017. 
16. R. Fletcher, N. I. M. Gould, S. Leyffer, P. L. Toint, and A. Wachter. Global convergence of trust-region SQP-filter algorithm for general nonlinear programming. SIAM J. Optimiz., 13:635-659, 2002.

17. R. Fletcher and S. Leyffer. Nonlinear programming without a penalty function. Math. Program., 91:239-269, 2002.

18. J. Gablonsky. DIRECT version 2.0 user guide. Technical Report CRSC-TR01-08, Center for Research in Scientific Computation, North Carolina State University, 2001.

19. M. L. N. Gonçalves, J. G. Melo, and L. F. Prudente. Augmented Lagrangian methods for nonlinear programming with possible infeasibility. J. Global Optim., 63:297-318, 2015.

20. C. C. Gonzaga, E. W. Karas, and M. Vanti. A globally convergent filter method for nonlinear programming. SIAM J. Optimiz., 14(3):646-669, 2003.

21. N. I. M. Gould, S. Leyffer, and P. L. Toint. A multidimensional filter algorithm for nonlinear equations and nonlinear least-squares. SIAM J. Optimiz., 15(1):17-38, 2004.

22. G. W. Greenwood and Q. J. Shu. Convergence in evolutionary programs with selfadaptation. Evol. Comput., 9(2):147-157, 2001.

23. Q. He and L. Wang. A hybrid particle swarm optimization with a feasibility-based rule for constrained optimization. Appl. Math. Comput., 186(2):1407-1422, 2007.

24. A.-R. Hedar and M. Fukushima. Derivative-free filter simulated annealing method for constrained continuous global optimization. J. Glob. Optim., 35(4):521-549, 2006.

25. E. W. Karas, A. P. Oening, and A. A. Ribeiro. Global convergence of slanting filter methods for nonlinear programming. Appl. Math. Comput., 200:486-500, 2008.

26. J. J. Liang, T. P. Runarsson, E. Mezura-Montes, M. Clerc, P. N. Suganthan, C. A. C. Coello, and K. Deb. Problem definitions and evaluation criteria for the CEC 2006 special session on constrained real-parameter optimization. Technical report, Nanyang Technological University, Singapore, 2006.

27. J. Long and S. Zeng. A new Filter-Levenberg-Marquart method with disturbance for solving nonlinear complementarity problems. Appl. Math. Comput., 216(2):677-688, 2010.

28. M. J. F. G. Macêdo, M. F. P. Costa, A. M. A. C. Rocha, and E. W. Karas. Combining filter method and dynamically dimensioned search for constrained global optimization. In O. Gervasi, B. Murgante, S. Misra, G. Borruso, C. Torre., A. Rocha, D. Taniar, B. Apduhan, E. Stankova, and A. Cuzzocrea, editors, Computational Science and Its Applications - ICCSA 2017: 17th International Conference, Trieste, Italy, July 3-6, 2017, Proceedings, Part III, pages 119-134. Springer International Publishing, 2017.

29. R. Mallipeddi and P. N. Suganthan. Problem definitions and evaluation criteria for the CEC 2010 competition on constrained real-parameter optimization. Technical report, Nanyang Technological University, Singapore, 2010.

30. L. Nuñez, R. G. Regis, and K. Varela. Accelerated random search for constrained global optimization assisted by radial basis function surrogates. J. Comput. Appl. Math., 340:276$295,2018$.

31. G. A. Periçaro, A. A. Ribeiro, and E. W. Karas. Global convergence of a general filter algorithm based on an efficiency condition of the step. Appl. Math. Comput., 219:95819597, 2013.

32. Y. G. Petalas, K. E. Parsopoulos, and M. N. Vrahatis. Memetic particle swarm optimization. Ann. Oper. Res., 156(1):99-127, 2007.

33. M. J. D. Powell. The theory of radial basis function approximation in 1990. In W. Light, editor, Advances in numerical analysis. Vol. 2. Wavelets, subdivision algorithms and radial basis functions, pages 105-210. Oxford University Press, 1992.

34. C. J. Price, , M. Reale, and B. L. Robertson. Stochastic filter methods for generally constrained global optimization. J. Global Optim., 65(3):441-456, 2016.

35. R. G. Regis. Convergence guarantees for generalized adaptive stochastic search methods for continuous global optimization. Eur. J. Oper. Res., 207:1187-1202, 2010.

36. R. G. Regis. Stochastic radial basis function algorithms for large-scale optimization involving expensive black-box objective and constraint functions. Comput. Oper. Res., 38:837-853, 2011.

37. R. G. Regis. Constrained optimization by radial basis function interpolation for highdimensional expensive black-box problems with infeasible initial points. Eng. Optimiz., 46:218-243, 2014.

38. R. G. Regis and C. A. Shoemaker. A stochastic radial basis function method for the global optimization of expensive functions. INFORMS J. Comput., 19(4):497-509, 2007.

39. R. G. Regis and C. A. Shoemaker. Combining radial basis function surrogates and dynamic coordinate search in high-dimensional expensive black-box optimization. Eng. Optimiz., 45:529-555, 2013. 
40. S. I. Resnick. A Probablity Path. Birkhauser, Boston, 1999.

41. A. A. Ribeiro, E. W. Karas, and C. C. Gonzaga. Global convergence of filter methods for nonlinear programming. SIAM J. Optimiz., 19(3):1231-1249, 2008.

42. A. M. A. C. Rocha, M. F. P. Costa, and E. M. G. P. Fernandes. A filter-based artificial fish swarm algorithm for constrained global optimization: theoretical and practical issues. J. Global Optim., 60:239-263, 2014.

43. A. M. A. C. Rocha and E. M. G. P. Fernandes. Hybridizing the electromagnetism-like algorithm with descent search for solving engineering design problems,. Int. J. Comput. Math., 86:1932-1946, 2009.

44. B. A. Tolson, M. Asadzadeh, and A. Zecchin. Hybrid discrete dynamically dimensioned search (HD-DDS) algorithm for water distribution system design optimization. Water Resour. Res., 45, 2009.

45. B. A. Tolson and C. A. Shoemaker. Dynamically dimensioned search algorithm for computationally efficient watershed model calibration. Water Resour. Res., 43, 2007.

46. M. Ulbrich, S. Ulbrich, and L. N. Vicente. A globally convergent primal-dual interior-point filter method for nonlinear programming. Math. Program., 100(2):379-410, 2004.

47. C.-Y. Wang and D. Li. Unified theory of augmented lagrangian methods for constrained global optimization. J. Glob. Optim., 44(3):433-458, 2008

48. X. Wang, Z. Zhu, S. Zuo, and Q. Huang. An SQP-filter method for inequality constrained optimization and its global convergence. Appl. Math. Comput., 217(24):10224-10230, 2011.

49. K. Q. Ye, W. Li, and A. Sudjianto. Algorithmic construction of optimal symmetric latin hypercube designs. J. Stat. Plan. Infer., 90(1):145-159, 2000 